\title{
Measuring gravitational waves from binary black hole coalescences. II. The waves' information and its extraction, with and without templates
}

\author{
Éanna É. Flanagan \\ Cornell University, Newman Laboratory, Ithaca, New York 14853-5001 \\ Scott A. Hughes \\ Theoretical Astrophysics, California Institute of Technology, Pasadena, California 91125
}

(Received 30 October 1997; published 2 April 1998)

\begin{abstract}
We discuss the extraction of information from detected binary black hole $(\mathrm{BBH})$ coalescence gravitational waves by the ground-based interferometers LIGO and VIRGO, and by the space-based interferometer LISA. We focus on the merger phase that occurs after the gradual inspiral and before the ringdown. Our results are (i) if numerical relativity simulations have not produced template merger waveforms before $\mathrm{BBH}$ events are detected, one can study the merger waves using simple band-pass filters. For BBHs smaller than about $40 M_{\odot}$ detected via their inspiral waves, the band-pass filtering signal-to-noise ratio indicates that the merger waves should typically be just barely visible in the noise for initial and advanced LIGO interferometers. (ii) We derive an optimized maximum-likelihood method for extracting a best-fit merger waveform from the noisy detector output; one "perpendicularly projects" this output onto a function space (specified using wavelets) that incorporates our (possibly sketchy) prior knowledge of the waveforms. An extension of the method allows one to extract the BBH's two independent waveforms from outputs of several interferometers. (iii) We propose a computational strategy for numerical relativists to pursue, if they successfully produce computer codes for generating merger waveforms, but if running the codes is too expensive to permit an extensive survey of the merger parameter space. In this case, for LIGO-VIRGO data analysis purposes, it would be advantageous to do a coarse survey of the parameter space aimed at exploring several qualitative issues and at determining the ranges of the several key parameters which we describe. (iv) A complete set of templates could be used to test the nonlinear dynamics of general relativity and to measure some of the binary's parameters via matched filtering. We estimate the number of bits of information obtainable from the merger waves (about 10-60 for LIGO-VIRGO, up to 200 for LISA), estimate the information loss due to template numerical errors or sparseness in the template grid, and infer approximate requirements on template accuracy and spacing. [S0556-2821(98)06208-0]
\end{abstract}

PACS number(s): 04.80.Nn, 04.25.Dm, 04.30.Db, 95.55.Ym

\section{INTRODUCTION AND SUMMARY}

\section{A. Gravitational waves from binary black holes}

With ground-based gravitational-wave observatories such as the Laser Interferometric Gravitational Wave Observatory (LIGO) [1], VIRGO [2], and GEO600 [3] expected to be taking data within the next few years, and with the spacebased Laser Interferometer Space Antenna (LISA) [4-6] in planning and development, much effort is currently going into understanding gravitational-wave sources and associated data analysis issues. One potentially interesting and important source is the coalescences of binary black holes (BBHs). Such systems will be detectable to large distances by groundbased interferometers (factors of the order of 10 further than binary neutron star systems) and over a wide range of masses. If the birthrates of BBH systems are not too low, they could be the most commonly detected type of compact binary gravitational-wave source.

The evolution of BBH systems and their emitted gravitational waves can be roughly divided into three epochs [7]: an adiabatic inspiral, in which the evolution is driven by radiation reaction, terminating roughly at the innermost stable circular orbit [8,9]; a violent, dynamic merger; and a final ringdown in which the emitted radiation is dominated by the $l$ $=m=2$ quasinormal mode of the final Kerr black hole.
Gravitational waves from the merger epoch could be rich with information about relativistic gravity in a highly nonlinear, highly dynamical regime which is poorly understood today.

Depending on the system's mass, some BBH coalescence events will be most easily detected by searching for the inspiral waves, others by searching for the ringdown, and others by searching for the merger. In paper I of this series [7], we analyzed the prospects for detecting $\mathrm{BBH}$ events using these three different types of searches, for initial and advanced LIGO interferometers and for LISA. Once a BBH event has been detected, the location of the three different phases of the waves in the data stream will be known to a fair approximation, although it will not necessarily be the case that all three phases will be detectable.

Waveform models or templates for the three epochs will be useful both for searches for BBH events using matched filtering, and also for interpreting and extracting information from the observed waveforms. At present, there is a reasonably good theoretical understanding of the waves generated during the inspiral and the ringdown $[7,10]$, whereas the merger is very poorly understood: no merger templates exist as yet. Theoretical understanding of merger dynamics will eventually come from numerical relativity. One rather large effort to compute the dynamics of BBH mergers is the 
American Grand Challenge Alliance, an NSF funded collaboration of physicists and computer scientists at eight institutions [11,12]; similar efforts are underway elsewhere. Modeling BBH mergers is an extremely difficult task; the numerical relativists who are writing codes for simulating BBH mergers are beset with many technical difficulties.

When the first BBH coalescences are detected, our theoretical understanding of $\mathrm{BBH}$ mergers could be in one of four possible states: (i) No information: supercomputer simulations have not yet successfully evolved any BBH mergers, and so no information about merger waves is available. (ii) Information limited in principle: some information about BBH mergers is available, but numerical relativists are unable to produce arbitrary merger templates. For example, supercomputer codes might only be able to simulate some special class of BBH mergers (e.g., those with vanishing initial spins or equal mass BBHs), or it could be that it is not possible to produce accurate waveforms, but more qualitative information about the merger (such as its duration) is available. (iii) Information limited in practice: accurate waveforms can be obtained for fully general BBH mergers, but each run of the codes to produce a template is so expensive in terms of computer time and cost that only a small number of representative template shapes can be computed and stored. (The total number of template shapes required to cover the entire range of behaviors of BBH mergers is likely to be in the range of thousands to millions or more.) (iv) Full information: a complete set of templates has been computed and is available for data analysis. This possibility seems rather unlikely in the time frame of the first detections of BBH coalescences.

Concomitant to these four states are three possible scenarios for data analysis of the waves from the merger epoch. The first possibility [corresponding to state (i) above] is that numerical computations provide no input to aid gravitational-wave data analysis. With no templates to guide the interpretation of the measured waveform, it will not be possible to obtain information about the $\mathrm{BBH}$ source or about strong-field general relativity from the merger waves. One's goal will simply be to measure as accurately as possible the merger waveform's shape. For this waveform shape measurement, one should make use of all possible prior information obtainable from analyses of the inspiral and/or ringdown signals, if they are detectable (see Sec. I B below).

Second [states (ii) and (iii) above], if only a few representative simulations and associated templates are available, one might simply perform a qualitative comparison between the measured waveform and templates in order to deduce qualitative information about the BBH source. For instance, simulations might demonstrate a strong correlation between the duration of the merger (in units of the total mass of the system) and the spins of the binary's black holes; a measurement of the merger's duration would then give some information about the binary's spins, without having to find a template that exactly matched the measured waveform. In this scenario, when reconstructing the merger waveform from the noisy data, one should use any prior information from the measured inspiral and/or ringdown waves, and in addition the prior information (for example the expected range of frequencies) one has about the merger waveforms' behaviors from representative supercomputer simulations.
The third scenario consists of matched filtering the data stream with merger templates in order to measure the parameters of the binary and to test general relativity. This will certainly be feasible if one has a complete set of merger templates [state (iv)]. It may also be feasible when information about BBH mergers is "limited in practice" [state (iii)]: it may be possible to perform several runs of the supercomputer code, concentrated in the appropriate small region of parameter space compatible with one's measurements from the inspiral and ringdown waves, in an effort to match the observed waveforms.

\section{B. What can be learned from $\mathrm{BBH}$ waves}

Different types of information will be obtainable from the three different phases of the gravitational-wave signal. If the inspiral and ringdown phases are strong enough to be measurable, they will be easier to analyze than the merger phase, and the information they yield via matched filtering will be used as "prior information" in attempting to analyze the merger. Matched filtering of the inspiral will allow measurements of the (redshifted) masses of the two black holes, the direction to the source, the arrival time, direction of orbital angular momentum, and orbital phase at some fiducial frequency, the luminosity distance to the source, and some information about the black holes' spins. See, for example, Refs. [13-18] for estimates of anticipated measurement accuracies for these parameters [19]. From the ringdown waves, one can measure the mass $M$ and dimensionless spin parameter $a$ of the final merged black hole, with an accuracy of roughly $[16,17]$

$$
\begin{gathered}
\Delta a \simeq \frac{6(1-a)^{1.06}}{(S / N)_{\text {ringdown }}}, \\
\frac{\Delta M}{M} \simeq \frac{2(1-a)^{9 / 20}}{(S / N)_{\text {ringdown }}},
\end{gathered}
$$

where $(S / N)_{\text {ringdown }}$ is the ringdown's measured matched filtering signal-to-noise ratio (SNR). (However, note that for low mass BBH events which are detected via their inspiral signals, the ringdown waves will be detectable only for $\sim 1 \%$ of the events [20]).

If merger templates are available, one could hope to use matched filtering to measure the system's parameters and to test general relativity. If one has no prior information about the detected BBH system, one would simply filter the merger data with all merger templates available, potentially a large number. However, if the inspiral and/or the ringdown signals have already been measured, some information of the type discussed in the previous paragraph will be available. In such cases the total number of merger templates needed will be reduced - one need consider only templates whose parameters are commensurate with the inspiral and ringdown measurements. Such inspiral and ringdown information will be invaluable if our understanding of the merger waves is "limited in practice," as discussed in Sec. I A.

The primary goal when one attempts to match a merger template with gravitational-wave data will be to provide a 
test of general relativity rather than to measure parameters. A good match between the measured waveform and a numerical template would constitute a strong test of general relativity in the most extreme of domains: highly nonlinear, rapidly dynamical, highly non-spherical spacetime warpage. It would also provide the oft-quoted unambiguous detection of black holes. (Such an unambiguous detection could also come from a measurement of quasi-normal ringing.) A close match between measured and predicted waveforms for $\mathrm{BBH}$ mergers might constrain theories of gravity that generalize general relativity. The inspiral portion of the waveform for neutron-star-neutron-star mergers will strongly constrain the dimensionless parameter $\omega$ of Brans-Dicke theory [21]. Unfortunately, the most theoretically natural class of generalizations of general relativity compatible with known experiments ("scalar-tensor theories" [22]) may not be constrained by BBH measurements, since black holes, unlike neutron stars, cannot have any scalar hair in such theories [23].

Matched filtering of the merger waves could also be useful in measuring some of the system's parameters, such as the total mass $M$ or the spin parameter $a$ of the final black hole [24]. These measurements could provide additional information about the source, over and above that obtainable from the inspiral and ringdown signals. For instance, in some cases the total mass of the system may be largely unconstrained from an inspiral measurement, while the ringdown may not be detectable; in such cases the total mass might be extractable from the merger waves.

\section{Extracting the waves' information: Our analyses, suggested tools, and results}

The principal purposes of this paper are (i) to suggest a data analysis method that can be used in the absence of templates to obtain from the noisy data stream a "best-fit", merger waveform shape and (ii) to provide input to numerical relativity simulations by deriving some requirements that numerical templates must satisfy in order to be as useful as possible for data analysis purposes and by highlighting the kinds of information that such simulations can provide, other than merger templates, that can aid $\mathrm{BBH}$ merger data analysis.

We first consider analysis of a detected merger without templates from numerical relativity. In this case, observers will likely resort to simple band-pass filters to study the merger waves. The first question to address in this context is whether the merger signal is likely to even be visible, that is, whether the signal will stand out above the background noise level in the band-pass filtered detector output. In Sec. III we estimate band-pass filtering signal to noise ratios (SNRs) for the merger waves using the results of paper I. We find that for BBHs that have been detected via their inspiral waves, these band-pass filtering SNRs are of order unity for initial and advanced LIGO interferometers; thus the merger signal will typically be just barely visible above the noise if at all. Only the somewhat rarer, close events will have easily visible merger signals. For LISA, by contrast, we estimate that band-pass filtering SNRs will typically be $\gtrsim 400$, and so the merger waves will be easily visible.

When templates are not available, one's goal will be to reconstruct as well as possible the merger waveform from the noisy data stream. In Sec. IV we use Bayesian statistics and the framework of maximum likelihood estimation to sketch an optimized method for performing such a reconstruction. The method is based on a "perpendicular projection" of the observed signal onto an appropriate function space that encodes all of our (possibly sketchy) prior knowledge about the waveforms. We argue that the best type of "basis functions", to use to specify this function space are wavelets, functions which allow simultaneous localization in time and frequency. We develop the reconstruction technique in detail using the language of wavelets, and also show that the operation of "perpendicular projection" onto the function space is a special case of Wiener optimal filtering. In Appendix A, we describe an extension of the method to a network of several gravitational-wave detectors, which allows one to reconstruct the two independent polarizations $h_{+}(t)$ and $h_{\times}(t)$ of the merger waves. This method for a network is an extension and generalization of a method previously suggested by Gürsel and Tinto [25].

Our waveform reconstruction algorithm comes in two versions: a simple version incorporating the above mentioned "perpendicular projection," described in Sec. IV A, and a more general and powerful version that allows one to build in more prior information, described in Sec. IV B. If one's prior information consists only of the signal's bandwidth, then the best-fit reconstructed waveform is just the band-pass filtered data stream. However, one can also build in as input to the method the expected duration of the signal, the fact that it must match up smoothly to the measured inspiral waveform, etc.; in such cases the reconstructed waveform differs from the band-pass filtered data stream.

In Sec. V, we discuss the types of information that representative supercomputer simulations could provide, short of providing a complete set of merger templates [i.e., in states (ii) and (iii) above], that would be useful for data analysis. Such qualitative information about BBH merger waveforms would be useful in two ways: as prior information for signal reconstruction and as a basis for comparisons with the reconstructed waveforms in order to make qualitative deductions about the $\mathrm{BBH}$ source, as mentioned above.

We turn next to issues concerning the use of numerical relativity templates in data analysis. Using matched filtering, templates can be used to make measurements of the binary's physical parameters (masses, vectorial spin angular momenta, etc.) which are independent of any such measurements from the inspiral and ringdown waves, and to make quantitative tests of general relativity. These measurements and tests will be possible with modest accuracy with LIGOVIRGO and with extremely high accuracy with LISA (for which the merger matched filtering SNRs are typically $\gtrsim 10^{4}$ [7]). To be useful for such purposes, the merger templates must satisfy certain accuracy requirements. In Sec. VI we derive an approximate accuracy criterion [Eq. (6.2)] that numerical relativists can use to ensure that the waveforms they produce are sufficiently accurate. This formula is derived from two requirements: first, that template inaccuracies cause a loss in event rate of no more than $3 \%$ when searching for merger waves with matched filtering, and second, when measuring the $\mathrm{BBH}$ parameters, that the systematic errors due to template inaccuracies be smaller than the statistical errors from detector noise. 
In Sec. VII we re-address the issue of template accuracy and also the issue of the spacing of templates in parameter space in the construction of a grid of templates, using the mathematical machinery of information theory. In information theory, a quantity called "information" can be associated with any measurement: it is simply the base 2 logarithm of the number of distinguishable measurement outcomes $[26,27]$. We specialize the notions of information theory to gravitational-wave measurements, and define two different types of information: (i) a "total" information $I_{\text {total }}$, the base 2 logarithm of the total number of waveform shapes that could have been distinguished by the measurement, and (ii) a "source" information $I_{\text {source }}$, the base 2 logarithm of the total number of waveform shapes that could have been distinguished by the measurement and that could have been generated by BBH mergers (i.e., the number of BBH sources that the measurement could have distinguished).

We give precise definitions of $I_{\text {total }}$ and $I_{\text {source }}$ [Eqs. (7.2) and (7.11)] in Sec. VII. In Appendix B, we derive simple analytic approximations for $I_{\text {total }}$ and $I_{\text {source }}$, expressing them in terms of the merger's matched filtering $\operatorname{SNR} \rho$, the number of independent data points, $\mathcal{N}_{\text {bins }}$, in the observed signal, and the number of parameters, $\mathcal{N}_{\text {param }}$, on which merger templates have a significant dependence. In Sec. VII C, we estimate the loss $\delta I_{\text {source }}$ in source information that would result from template inaccuracies [Eq. (7.20)]; demanding that $\delta I_{\text {source }} \leqslant 1$ then allows us to re-derive the criterion for the template accuracy requirements obtained in Sec. VI. We also estimate the loss in information $\delta I_{\text {source }}$ that would result from having insufficiently closely spaced templates in a template grid [Eq. (7.24)], and we deduce an approximate criterion for how closely templates must be spaced.

\section{NOTATION AND CONVENTIONS}

In this section we introduce some notation that will be used throughout the paper. We use geometrized units in which Newton's gravitational constant $G$ and the speed of light $c$ are unity. For any function of time $a(t)$, we will use a tilde to represent that function's Fourier transform:

$$
\widetilde{a}(f)=\int_{-\infty}^{\infty} d t e^{2 \pi i f t} a(t) .
$$

The output strain amplitude $s(t)$ of a detector can be written

$$
s(t)=h(t)+n(t),
$$

where $h(t)$ is the gravitational wave signal and $n(t)$ is the detector noise. Throughout this paper we will assume, for simplicity, that the noise is stationary and Gaussian. The statistical properties of the noise determine an inner product on the space of waveforms $h(t)$, given by

$$
\left(h_{1} \mid h_{2}\right)=4 \operatorname{Re} \int_{0}^{\infty} d f \frac{\widetilde{h_{1}}(f) * \widetilde{h_{2}}(f)}{S_{h}(f)} ;
$$

see, for example, Refs. $[28,13]$. In Eq. $(2.3), S_{h}(f)$ is the one-sided power spectral density of strain noise $n(t)$ [29].

For any waveform $h(t)$, the matched filtering $\operatorname{SNR} \rho$ is given by

$$
\rho^{2}=(h \mid h)=4 \int_{0}^{\infty} d f \frac{|\widetilde{h}(f)|^{2}}{S_{h}(f)} .
$$

On several occasions we shall be interested in finite stretches of data of length $T$, represented as a vector of numbers instead of as a continuous function. If $\Delta t$ is the sampling time, this vector is

$$
\mathbf{s}=\left(s^{1}, \ldots, s^{\mathcal{N}_{\text {bins }}}\right)
$$

where $\mathcal{N}_{\text {bins }}=T / \Delta t, \quad s^{j}=s\left[t_{\text {start }}+(j-1) \Delta t\right], \quad 1 \leqslant j \leqslant \mathcal{N}_{\text {bins }}$, and $t_{\text {start }}$ is the starting time. The quantity $\mathcal{N}_{\text {bins }}$ is the number of independent real data points (number of bins) in the measured signal. The gravitational wave signal $h(t)$ and the noise $n(t)$ can similarly be represented in this way, so that $\mathbf{s}=\mathbf{h}+\mathbf{n}$. We adopt the geometrical viewpoint of Dhurandhar and Schutz [30], regarding $\mathbf{S}$ as an element of an abstract vector space $V$ of dimension $\mathcal{N}_{\text {bins }}$, and the sample points $s^{j}$ as the components of $\mathbf{s}$ on a time domain basis $\left\{\mathbf{e}_{1}, \ldots, \mathbf{e}_{\mathcal{N}_{\text {bins }}}\right\}$ of $V$ :

$$
\mathbf{s}=\sum_{j=1}^{\mathcal{N}_{\text {bins }}} s^{j} \mathbf{e}_{j} .
$$

Taking a finite Fourier transform of the data stream can be regarded as a change of basis of $V$. Thus, a frequency domain basis $\left\{\mathbf{d}_{k}\right\}$ of $V$ is given by the finite Fourier transform

$$
\mathbf{d}_{k}=\sum_{j=1}^{\mathcal{N}_{\text {bins }}} \mathbf{e}_{j} \exp \left\{2 \pi i j k / \mathcal{N}_{\text {bins }}\right\},
$$

where $-\left(\mathcal{N}_{\text {bins }}-1\right) / 2 \leqslant k \leqslant\left(\mathcal{N}_{\text {bins }}-1\right) / 2$. The corresponding frequencies $f_{k}=k / T$ run from $-1 /(2 \Delta t)$ to $1 /(2 \Delta t)$ [31].

More generally, if we band-pass filter the data stream down to a frequency interval of length $\Delta f$, then a stretch of band-pass filtered data of duration $T$ will have

$$
\mathcal{N}_{\text {bins }}=2 T \Delta f
$$

independent real data points. In this case also we regard the set of all such stretches of data as an abstract linear space $V$ of dimension $\mathcal{N}_{\text {bins }}$.

On an arbitrary basis of $V$, we define the matrices $\Gamma_{i j}$ and $\sum^{i j}$ by

$$
\begin{aligned}
& \left\langle n^{i} n^{j}\right\rangle \equiv \Sigma^{i j}, \\
& \Gamma_{i j} \Sigma^{j k}=\delta_{k}^{i} ;
\end{aligned}
$$

i.e., the matrices $\boldsymbol{\Gamma}$ and $\boldsymbol{\Sigma}$ are inverses of each other. In Eq. (2.9) the angular brackets mean expected value. On the time domain basis $\left\{\mathbf{e}_{1}, \ldots, \mathbf{e}_{\mathcal{N}_{\text {bins }}}\right\}$ we have

$$
\Sigma^{j k}=C_{n}\left(t_{j}-t_{k}\right)
$$

where $t_{j}=t_{\text {start }}+(j-1) \Delta t$, and $C_{n}(\tau)=\langle n(t) n(t+\tau)\rangle$ is the noise correlation function given by

$$
C_{n}(\tau)=\int_{0}^{\infty} d f \cos [2 \pi f \tau] S_{h}(f) .
$$

We define an inner product on the space $V$ by 


$$
\left(\mathbf{h}_{1} \mid \mathbf{h}_{2}\right)=\Gamma_{i j} h_{1}^{i} h_{2}^{j} .
$$

This is a discrete version of the inner product (2.3): the two inner products coincide in the limit $\Delta t \rightarrow 0$, for waveforms which vanish outside of the time interval of length $T$ [16].

Throughout this paper we shall use interchangeably the notations $h(t)$ and $\mathbf{h}$ for a gravitational waveform. We shall also for the most part not need to distinguish between the inner products (2.3) and (2.13). Some generalizations of these notations and definitions to a network of several detectors are given in Appendix A.

For any detector output $\mathbf{s}=\mathbf{h}+\mathbf{n}$, we define

$$
\rho(\mathbf{s}) \equiv \sqrt{(\mathbf{s} \mid \mathbf{s})},
$$

which we call the magnitude of the stretch of data $\mathbf{s}$. From Eqs. (2.9) and (2.13) it follows that

$$
\left\langle\rho(\mathbf{s})^{2}\right\rangle=\rho^{2}+\mathcal{N}_{\text {bins }},
$$

where $\rho^{2}$ is the matched filtering SNR squared (2.4) of the signal $\mathbf{h}$, and that

$$
\sqrt{\left\langle\left[\Delta \rho(\mathbf{s})^{2}\right]^{2}\right\rangle}=\sqrt{4 \rho^{2}+2 \mathcal{N}_{\text {bins }}},
$$

where $\Delta \rho(\mathbf{s})^{2} \equiv \rho(\mathbf{s})^{2}-\left\langle\rho(\mathbf{s})^{2}\right\rangle$. Thus, the magnitude $\rho(\mathbf{s})$ is approximately the same as the matched filtering SNR $\rho$ in the limit $\rho \gg \sqrt{\mathcal{N}_{\text {bins }}}$ (large SNR squared per frequency bin), but is much larger than $\rho$ when $\rho \ll \sqrt{\mathcal{N}_{\text {bins. }} \text {. The quantity } \rho(\mathbf{s})}$ will be of most use in our information theory calculations in Sec. VII and Appendix B.

The space $V$ equipped with the inner product (2.13) forms a Euclidean vector space. We will also be concerned with sets of gravitational waveforms $\mathbf{h}(\boldsymbol{\theta})$ that depend on a finite number $n_{p}$ of parameters $\boldsymbol{\theta}=\left(\theta^{1}, \ldots, \theta^{n}\right)$. For example, inspiral waveforms form a set of this type, where $\boldsymbol{\theta}$ are the parameters describing the binary source. We will denote by $\mathcal{S}$ the manifold of signals $\mathbf{h}(\boldsymbol{\theta})$, which is a submanifold of dimension $n_{p}$ of the vector space $V$. We will adopt the convention that Roman indices $i, j, k, \ldots$ will run from 1 to $\mathcal{N}_{\text {bins }}$, and that a symbol such as $v^{i}$ will denote some vector in the space $V$. Greek indices $\alpha, \beta, \gamma$ will run from 1 to $n_{p}$, and a vector $v^{\alpha}$ will denote a vector field on the manifold $\mathcal{S}$. The inner product (2.13) induces a natural Riemannian metric on the manifold $\mathcal{S}$ given by

$$
d s^{2}=\left(\frac{\partial \mathbf{h}}{\partial \theta^{\alpha}} \mid \frac{\partial \mathbf{h}}{\partial \theta^{\beta}}\right) d \theta^{\alpha} d \theta^{\beta} .
$$

We shall denote this metric by $\Gamma_{\alpha \beta}$ and its inverse by $\Sigma^{\alpha \beta}$, relying on the index alphabet to distinguish these quantities from the quantities (2.9) and (2.10). For more details on this geometric picture, see, for example, Ref. [13].

We shall use the word detector to refer to either a single interferometer or a resonant mass antenna and the phrase detector network to refer to a collection of detectors operated in tandem. Note that this terminology differs from that adopted in, for example, Ref. [28], where a detector network is called a detector.

Finally, we will use boldfaced vectors like a to denote either vectors in three dimensional space, or vectors in the $\mathcal{N}_{\text {bins }}$-dimensional space $V$, or vectors in the $n_{p}$ dimensional space of signal parameters. In Appendix A, we will use arrowed vectors $(\vec{a})$ to denote elements of the linear space of the output of a detector network.

\section{ANALYSIS OF MERGER WAVES WITHOUT TEMPLATES: VISIBILITY OF THE MERGER AFTER BAND-PASS FILTERING}

We first consider merger wave data analysis when matched filtering is not possible. One's primary goal in this case will be to reconstruct a "best-guess" estimate of the merger waveform [32] from the measured data. If some (perhaps very few) supercomputer templates are available, it may then be possible to interpret the reconstructed waveform and obtain some qualitative information about the source.

One very simple procedure that could be used to estimate the waveform shape is simply to band-pass filter the data stream according to our prior prejudice about the frequency band of the merger waves. However, even after such bandpass filtering, the merger signal may be dominated by detector noise and may not be visible.

In this section, we estimate the visibility of the merger signal after band-pass filtering by calculating band-pass filtering SNRs using the results of Ref. [7]. A signal will be visible if its band-pass filtering SNR is large compared to unity [7]. We consider only signals that are detected via their inspiral waves, i.e., low-mass BBH systems. We first consider the visibility of the last few cycles of the inspiral. By continuity, one might expect that if the last few inspiral cycles are visible, then at least the early part of the merger signal will be as well. We then consider the visibility of the merger signal itself.

\section{A. Visibility of inspiral waveform}

If a BBH event has been detected via its inspiral signal, it follows that the matched filtering inspiral SNR must be $\gtrsim 6$ [33]. It does not follow, however, that the inspiral is visible in the data stream. For neutron-star-neutron-star binaries the reverse is usually the case: the amplitude of the signal is less than the noise, and the signal would be invisible without matched filtering.

The dominant harmonic of the inspiral waveform can be written as

$$
h(t)=h_{\mathrm{amp}}(t) \cos [\Phi(t)],
$$

where the amplitude $h_{\text {amp }}(t)$ and instantaneous frequency $f(t)$ [given by $2 \pi f(t)=d \Phi / d t$ ] are slowly evolving. For such waveforms, the SNR squared obtained using band-pass filtering is approximately given by the matched filtering SNR squared per cycle [cf. Eq. (2.10) of Ref. [7]]:

$$
\begin{aligned}
\left(\frac{S}{N}\right)_{\text {band-pass }}^{2} & \approx\left(\frac{S}{N}\right)_{\text {matched, per cycle }}^{2} \\
& =\left[\frac{h_{\text {amp }}[t(f)]}{h_{\text {rms }}(f)}\right]^{2}
\end{aligned}
$$

In Eq. (3.2), $t(f)$ denotes the time at which the frequency is $f$, and $h_{\mathrm{rms}}(f) \equiv \sqrt{f S_{h}(f)}$. Note that the band-pass filtering SNR (3.2) is evaluated at a specific frequency; when one 
discusses matched filtering SNRs, an integral over a frequency band has been performed. We next insert the value of $h_{\mathrm{amp}}[t(f)]^{2}$ for the leading-order approximation to the inspiral waves and take an rms average over source orientations and polarizations $[34,7]$, which yields

$$
\left(\frac{S}{N}\right)_{\text {band-pass }}^{2}=\frac{4 \pi^{4 / 3}[(1+z) M]^{10 / 3} f^{4 / 3}}{25 D(z)^{2} h_{\mathrm{rms}}(f)^{2}} .
$$

Here, $M$ is the binary's total mass, $z$ its cosmological redshift, and $D(z)$ its luminosity distance. We have also specialized to equal masses.

In Eq. (4.1) of Ref. [7] we introduced an analytic formula for a detector's noise spectrum, which, by specialization of its parameters, could describe to a good approximation either an initial LIGO interferometer, an advanced LIGO interferometer, or a space-based LISA interferometer. We now insert that formula into Eq. (3.2), and specialize to the frequency

$$
f=f_{\text {merge }}=\frac{\gamma_{m}}{(1+z) M},
$$

where $\gamma_{m}=0.02$. The frequency $f_{\text {merge }}$ is approximately the location of the transition from inspiral to merger, as estimated in Ref. [7]. This yields

$$
\left(\frac{S}{N}\right)_{\text {band-pass }}^{2} \approx \frac{4 \pi^{4 / 3}[(1+z) M]^{5} \gamma_{m}^{-5 / 3} \alpha^{3} f_{m}^{3}}{5 D(z)^{2} h_{m}^{2}},
$$

where $\alpha, h_{m}$ and $f_{m}$ are the parameters used in Ref. [7] to describe the noise curve. Equation (3.5) is valid only when the redshifted mass $(1+z) M$ is smaller than $\gamma_{m} / \alpha f_{m}$.

For initial LIGO interferometers, appropriate values of $h_{m}, f_{m}$ and $\alpha$ are given in Eq. (4.2) of Ref. [7]. Inserting these values into Eq. (3.5) gives

$$
\left(\frac{S}{N}\right)_{\text {band-pass }} \sim 1.1\left[\frac{200 \mathrm{Mpc}}{D(z)}\right]\left[\frac{(1+z) M}{20 M_{\odot}}\right]^{5 / 2} .
$$

This result is valid for $(1+z) M \leq 18 M_{\odot}$. Now, the SNR obtained by matched filtering the inspiral signal is approximately [7]

$$
\left(\frac{S}{N}\right)_{\text {matched }} \sim 2.6\left[\frac{200 \mathrm{Mpc}}{D(z)}\right]\left[\frac{(1+z) M}{20 M_{\odot}}\right]^{5 / 6},
$$

and the SNR (3.7) must be $\gtrsim 6$ [33], since, by assumption, the inspiral has been detected. By eliminating the luminosity distance $D(z)$ between Eqs. (3.6) and (3.7) we find that the band-pass filtering SNR for the last few cycles of inspiral for detected binaries satisfies

$$
\left(\frac{S}{N}\right)_{\text {band-pass }} \geq 2.5\left[\frac{(1+z) M}{20 M_{\odot}}\right]^{5 / 3} .
$$

Therefore, the last few cycles of the inspiral should be individually visible above the noise for $\mathrm{BBH}$ events with $5 M_{\odot}$ $\lesssim M \lessgtr 20 M_{\odot}$ detected by initial LIGO interferometers.

We now repeat the above calculation with the values of $h_{m}, f_{m}$, and $\alpha$ appropriate for advanced LIGO interferom- eters, given in Eq. (4.3) of Ref. [7]. The band-pass filtering SNR for advanced interferometers is

$$
\left(\frac{S}{N}\right)_{\text {band-pass }} \sim 1.6\left[\frac{1 \mathrm{Gpc}}{D(z)}\right]\left[\frac{(1+z) M}{20 M_{\odot}}\right]^{5 / 2},
$$

and the SNR obtained by matched filtering the inspiral signal is

$$
\left(\frac{S}{N}\right)_{\text {matched }} \sim 16\left[\frac{1 \mathrm{Gpc}}{D(z)}\right]\left[\frac{(1+z) M}{20 M_{\odot}}\right]^{5 / 6},
$$

for $(1+z) M \leqslant 37 M_{\odot}$ [7]. With the assumption that $(S / N)_{\text {matched }} \gtrsim 6$, we find

$$
\left(\frac{S}{N}\right)_{\text {band-pass }} \geq 0.6\left[\frac{(1+z) M}{20 M_{\odot}}\right]^{5 / 3}
$$

for $(1+z) M \leqslant 37 M_{\odot}$. Thus the last few cycles of BBH inspirals with $(1+z) M \leqslant 37 M_{\odot}$ should typically be just barely visible above the noise for advanced LIGO interferometers, depending on the binary's total mass.

Although we do not explore here larger mass BBHs, in many cases for these systems also the last few cycles of inspiral will be visible; this can be seen by combining Eq. (3.2) with Figs. 4 and 5 of Ref. [7].

For LISA, Eq. (3.5) combined with Eq. (4.4) of Ref. [7] yields

$$
\left(\frac{S}{N}\right)_{\text {band-pass }} \sim 400\left[\frac{1 \mathrm{Gpc}}{D(z)}\right]\left[\frac{(1+z) M}{10^{5} M_{\odot}}\right]^{5 / 2}
$$

for $(1+z) M \lessgtr 10^{5} M_{\odot}$, with larger values for $10^{5} M_{\odot} \lesssim(1$ $+z) M \lessgtr 3 \times 10^{7} M_{\odot}$. Individual cycles of inspiral should be clearly visible with LISA.

\section{B. Visibility of merger waveform}

Consider now the merger waveform itself. In Ref. [7] we showed that

$$
\left(\frac{S}{N}\right)_{\text {band-pass, merger }} \approx \frac{1}{\sqrt{\mathcal{N}_{\text {bins }}}}\left(\frac{S}{N}\right)_{\text {matched, merger }},
$$

where $\mathcal{N}_{\text {bins }}=2 T \Delta f$ is as discussed in Sec. II. We also estimated [Eq. (3.26) of Ref. [7]] that, for the merger waves,

$$
\sqrt{\mathcal{N}_{\text {bins }}} \sim 5 \text {, }
$$

although there is a large uncertainty in this estimate.

Consider the band-pass filtering SNR for the merger for events that have been detected via matched filtering of the inspiral. For initial LIGO interferometers, combining Eqs. (B4) and (B10) of Ref. [7] Eqs. (3.13) and (3.14), and the threshold for detection [33],

$$
\left(\frac{S}{N}\right)_{\text {matched, inspiral }} \geq 6 \text {, }
$$

yields 


$$
\left(\frac{S}{N}\right)_{\text {band-pass, merger }} \geq 0.8\left[\frac{(1+z) M}{20 M_{\odot}}\right]^{5 / 3}
$$

for $(1+z) M \leqslant 18 M_{\odot}$. Repeating this analysis for advanced LIGO interferometers [using Eqs. (B5) and (B11) of Ref. [7]] yields

$$
\left(\frac{S}{N}\right)_{\text {band-pass, merger }} \geq 0.2\left[\frac{(1+z) M}{20 M_{\odot}}\right]^{5 / 3}
$$

for $(1+z) M \leqslant 37 M_{\odot}[35]$.

The SNR values (3.16) and (3.17) indicate that for typical inspiral-detected BBH systems with $M \lessgtr 20 M_{\odot}$ (initial interferometers) or $M \$ 40 M_{\odot}$ (advanced interferometers), the merger signal will not be easily visible in the noise, and that only relatively rare, nearby events will have easily visible merger signals. This conclusion is somewhat tentative because of the uncertainty in the estimates of $\mathcal{N}_{\text {bins }}$ and of the energy spectra discussed in Ref. [7]. Also the actual visibility will probably vary considerably from event to event. However, our crude visibility argument suggests that the prospects for accurately recovering the merger waveform are good only for the stronger detected merger signals.

This conclusion only applies to low mass BBH systems which are detected via their inspiral waves. For higher mass systems which are detected directly via their merger and/or ringdown waves, the merger signal should be visible above the noise after appropriate band-pass filtering (cf. Figs. 4 and 5 of [7], dividing the matched filtering SNRs presented there by $\sqrt{\mathcal{N}_{\text {bins }}} \sim 5$ ). Moreover, most merger events detected by LISA will have band-pass filtering SNRs $\gg 1$, as can be seen from Fig. 6 of Ref. [7], and thus should be easily visible.

\section{ANALYSIS OF MERGER WAVES WITHOUT TEMPLATES: A METHOD OF EXTRACTING A BEST-GUESS WAVEFORM FROM THE NOISY DATA STREAM}

In the absence of templates we would like to reconstruct from the data a best-guess estimate of the merger waveform. Any waveform-reconstruction method should use all available prior knowledge about the waveform. We will hopefully know from representative simulations and perhaps from the measured inspiral or ringdown the following: the approximate starting time of the merger waveform, the fact that it starts off strongly (smoothly joining on to the inspiral) and eventually dies away in quasinormal ringing, and its approximate bandwidth and duration. When both the inspiral and the ringdown are strong enough to be detectable, the duration of the merger waveform will be fairly well known, as will the frequency $f_{\text {qnr }}$ of the ringdown onto which the merger waveform must smoothly join.

In this section, we suggest a method for reconstructing the waveform which uses such prior information, based on the technique of maximum likelihood estimation [36,37]. We shall describe this method in the context of a single detector. However, in a few years there will be in operation a network of detectors (both interferometers [1-3] and resonant mass antennae), and from the outputs of these detectors one would like to reconstruct the two polarization components $h_{+}(t)$ and $h_{\times}(t)$ of the merger waves. In Appendix A we extend this section's waveform-estimation method to an arbitrary number of detectors, which yields a method of reconstructing the two waveforms $h_{+}(t)$ and $h_{\times}(t)$.

The use of maximum likelihood estimators has been discussed extensively by many authors in the context of gravitational waves of a known functional form, depending only on a few parameters $[28,13,14,38,39]$. Here we consider their application to wave bursts of largely unknown shape. The resulting data analysis methods which we derive are closely related mathematically to the methods discussed previously $[28,13,14,38,39]$, but are considerably different in operational terms and in implementation.

\section{A. Derivation of data analysis method}

Suppose our prior information includes the fact that the merger waveform lies inside some time interval of duration $T$ and inside some frequency interval of length $\Delta f$. We define $\mathcal{N}_{\text {bins }}=2 T \Delta f$; cf. Sec. II above. We assume that we are given a stretch of data of duration $T^{\prime}>T$ and with sampling time $\Delta t<1 /(2 \Delta f)$. These data lie in a linear space $V$ of dimension

$$
\mathcal{N}_{\text {bins }}^{\prime}=T^{\prime} / \Delta t
$$

Thus, $\mathcal{N}_{\text {bins }}^{\prime}$ is the number of independent data points, and $\mathcal{N}_{\text {bins }}$ is the number of independent data points in that subset of the data which we expect to contain the merger signal. Note that these definitions modify the conventions of Sec. II, where the dimension of $V$ was denoted $\mathcal{N}_{\text {bins }}$; we will use, unmodified, the other conventions of Sec. II.

In our analysis, we will allow the basis of the vector space $V$ to be arbitrary. However, we will occasionally specialize to the time-domain and frequency-domain bases discussed in Sec. II. We will also consider wavelet bases. Wavelet bases can be regarded as any set of functions $w_{i j}(t)$ such that $w_{i j}(t)$ is approximately localized in time at the time $t_{i}$ $=t_{\text {start }}+\left(i / n_{T}\right) T^{\prime}$ and in frequency at the frequency $f_{j}$ $=\left(j / n_{F}\right)(\Delta t)^{-1}$; their advantage is that they simultaneously encode time domain and frequency domain information. The index $i$ runs from 1 to $n_{T}$ and $j$ from $-\left(n_{F}-1\right) / 2$ to $\left(n_{F}\right.$ $-1) / 2$. Clearly the number of frequency bins $n_{F}$ and the number of time bins $n_{T}$ must satisfy $n_{T} n_{F}=\mathcal{N}_{\text {bins }}^{\prime}$, but otherwise they can be arbitrary; typically $n_{T} \sim n_{F} \sim \sqrt{\mathcal{N}_{\text {bins. }}^{\prime}}$. Also, the functions $w_{i j}$ usually all have the same shape,

$$
w_{i j}(t) \propto \varphi\left[f_{j}\left(t-t_{i}\right)\right],
$$

for some function $\varphi$. For our considerations, the shape of $\varphi$ is not of critical importance. Note that families of wavelets discussed in the literature are often overcomplete; here we are considering bases of the vector space $V$, which by definition are simply complete.

Let $p^{(0)}(\mathbf{h})$ be the probability distribution function (PDF) that summarizes our prior information about the waveform. A standard Bayesian analysis shows that the PDF of $\mathbf{h}$ given the measured data stream $\mathbf{s}$ is $[28,16]$

$$
p(\mathbf{h} \mid \mathbf{s})=\mathcal{K} p^{(0)}(\mathbf{h}) \exp \left[-\Gamma_{i j}\left(h^{i}-s^{i}\right)\left(h^{j}-s^{j}\right) / 2\right],
$$

where the matrix $\Gamma_{i j}$ is defined in Eq. (2.10) and $\mathcal{K}$ is a normalization constant. In principle this PDF gives complete 
information about the measurement. Maximizing the PDF gives the maximum likelihood estimator for the merger waveform $\mathbf{h}$. This estimator, $\mathbf{h}(\mathbf{s})$, will in general will be some non-linear function of $\mathbf{s}$. The effectiveness of the resulting waveform estimator will depend on how much prior information about the waveform shape can be encoded in the prior $\operatorname{PDF} p^{(0)}$.

One of the simplest possibilities is to take $p^{(0)}$ to be concentrated on some linear subspace $U$ of the space $V$ and to be approximately constant inside this subspace. A multivariate Gaussian with widths very small in some directions and very broad in others would accomplish this to a good approximation. For such choices of the prior PDF $p^{(0)}$, the resulting maximum likelihood estimator [the function $\mathbf{h}=\mathbf{h}(\mathbf{s})$ that maximizes the PDF (4.3)] is simply the perpendicular projection $P_{U}$ of $\mathbf{s}$ into $U$ :

$$
\mathbf{h}_{\text {best-fit }}(\mathbf{s})=P_{U}(\mathbf{s}),
$$

where

$$
P_{U}(\mathbf{s}) \equiv \sum_{i, j=1}^{n_{U}} u^{i j}\left(\mathbf{u}_{j} \mid \mathbf{s}\right) \mathbf{u}_{i}
$$

Here, $\mathbf{u}_{1}, \ldots, \mathbf{u}_{n_{U}}$ is an arbitrary basis of $U, n_{U}$ is the dimension of $U, u^{i j} u_{j k}=\delta_{j}^{i}$ and $u_{j k}=\left(\mathbf{u}_{j} \mid \mathbf{u}_{k}\right)$.

The method of filtering (4.4) is a special case of Wiener optimal filtering: it is equivalent to matched filtering with templates consisting of linear combinations of the basis functions $\mathbf{u}_{i}$. (The equivalence between maximum likelihood estimation and Wiener optimal filtering in more general contexts has been shown by Echeverria [40].) To show this, define a family of template waveforms that depends on parameters $a_{1}, \ldots, a_{n_{U}}$ by

$$
h\left(t ; a_{j}\right)=\sum_{j=1}^{n_{U}} a_{j} u_{j}(t),
$$

where $u_{j}(t)$ are the functions of time corresponding to the basis elements $\mathbf{u}_{j}$ of $U$. Now the SNR for any template $h(t)$ with the data stream $s(t)$ is

$$
\frac{S}{N}[h(t)] \equiv \frac{(\mathbf{h} \mid \mathbf{s})}{\sqrt{(\mathbf{h} \mid \mathbf{h})}} .
$$

The best-fit signal given by the optimal filtering method is the template which maximizes the SNR (4.7), i.e., the template $h\left(t ; \hat{a}_{j}\right)$ such that

$$
\frac{S}{N}\left[h\left(t ; \hat{a}_{j}\right)\right]=\max _{a_{1}, \ldots, a_{n_{U}}} \frac{S}{N}\left[h\left(t ; a_{j}\right)\right] .
$$

From Eqs. (4.5)-(4.7) it follows that $P_{U}(\mathbf{s})=h\left(t ; \hat{a}_{j}\right)$. Thus, computing the perpendicular projection (4.5) of $\mathbf{s}$ into $U$ is equivalent to matched filtering with the template family (4.6).

To summarize, the maximum likelihood estimator (4.4) gives a general procedure for specifying a filtering algorithm adapted to a given linear subspace $U$ of the space of signals $V$. We now discuss some general issues regarding the choice of $U$. At the very least, we would like our choice to effect truncation of the measured data stream in both the time and frequency domains, down to the intervals of time and frequency in which we expect the merger waveform to lie. Because of the uncertainty principle, such a truncation cannot be done exactly. Moreover, for fixed specific intervals of time and frequency, there are different, inequivalent ways of approximately truncating the signal to these intervals [41]. The differences between the inequivalent methods are essentially due to aliasing effects. Such effects cannot always be neglected in the analysis of merger waveforms, because the duration $T \sim 10 M-100 M$ [7] of the waveform is probably only a few times larger than the reciprocal of the highest frequency of interest.

The simplest method of truncating in frequency, bandpass filtering, is to a good approximation a projection of the type (4.4) that we are considering. Let $\mathbf{d}_{k}[\mathrm{cf}$. Eq. (2.7)] be a frequency domain basis of $V$. For a given frequency interval $\left[f_{\text {char }}-\Delta f / 2, f_{\text {char }}+\Delta f / 2\right]$, let $U$ be the subspace of $V$ spanned by the elements $\mathbf{d}_{j}$ with $\left|f_{\text {char }}-f_{j}\right|<\Delta f / 2$, i.e., the span of the basis elements that correspond to the given frequency interval. Then the projection operation $P_{U}$ is to a moderate approximation just the band-pass filter:

$$
P_{U}\left[\sum_{j=1}^{\mathcal{N}_{\text {bins }}^{\prime}} s^{j} \mathbf{d}_{j}\right] \approx \sum^{\prime}=s^{j} \mathbf{d}_{j}
$$

where the notation $\Sigma^{\prime}$ means that the sum is taken only over the appropriate range of frequencies. The relation (4.9) follows from the fact that the basis $\mathbf{d}_{j}$ is approximately orthogonal with respect to the noise inner product (2.13): different frequency components of the noise are statistically independent up to small aliasing corrections of the order of $\sim 1 /\left(f_{\text {char }} T^{\prime}\right)$. Thus, if our a priori information is that the signal lies within a certain frequency interval, then the maximum likelihood estimate of the signal is approximately given by passing the data stream through a band-pass filter.

Truncating in the time domain, on the other hand, is not a projection of the type (4.4). If our prior information is that the signal vanishes outside a certain interval of time, then simply discarding the data outside of this interval will not give the maximum likelihood estimate of the signal. This is because of statistical correlations between sample points just inside and just outside of the time interval: the measured data stream outside the interval gives information about what the noise inside the interval is likely to be. These correlation effects become unimportant in the limit $T f_{\text {char }} \rightarrow \infty$, but for BBH merger signals $T f_{\text {char }}$ is probably $\lesssim 20$ [7]. The correct maximum likelihood estimator of the waveform, when our prior information is that the signal vanishes outside of a certain time interval, is given by Eq. (4.5) with the basis $\left\{\mathbf{u}_{1}, \ldots, \mathbf{u}_{n_{U}}\right\}$ replaced by the appropriate subset of the timedomain basis $\left\{\mathbf{e}_{1}, \ldots, \mathbf{e}_{\mathcal{N}_{\text {bins }}^{\prime}}\right\}$.

Our suggested choice of subspace $U$ and corresponding specification of a filtering method is as follows. Pick a wavelet basis $\mathbf{w}_{i j}$ of the type discussed above. (The filtering method will depend only weakly on which wavelet basis is chosen.) Then, the subspace $U$ is taken to be the span of a suitable subset of this wavelet basis, chosen according to our 
prior information about the bandwidth and duration of the signal. The dimension of $U$ will be $n_{U}=\mathcal{N}_{\text {bins }}=2 T \Delta f$.

In more detail, the filtering method would work as follows. First, band-pass filter the data stream and truncate it in time, down to intervals of frequency and time that are several times larger than are ultimately required, in order to reduce the number of independent data points $\mathcal{N}_{\text {bins }}^{\prime}$ to a manageable number. Second, for the wavelet basis $\mathbf{w}_{i j}$ of this reduced data set, calculate the matrix $w_{i j i^{\prime} j^{\prime}}=\left(\mathbf{w}_{i j} \mid \mathbf{w}_{i^{\prime} j^{\prime}}\right)$. Recall that the index $i$ corresponds to a time $t_{i}$ and the index $j$ to a frequency $f_{j}$ [cf. the discussion preceding Eq. (4.2)]. Third, pick out the sub-block $\bar{w}_{i j i^{\prime} j^{\prime}}$ of the matrix $w_{i j i^{\prime} j^{\prime}}$ for which the times $t_{i}$ and $t_{i^{\prime}}$ and frequencies $f_{j}$ and $f_{j^{\prime}}$ lie in the required intervals. Invert this matrix to obtain $\bar{w}^{i j i^{\prime} j^{\prime}}$. The best-fit waveform is then given by

$$
\mathbf{h}_{\text {best-fit }}=\sum_{i j}^{\prime} \sum_{i^{\prime} j^{\prime}}{ }^{\prime} \bar{w}^{i j i^{\prime} j^{\prime}}\left(\mathbf{s} \mid \mathbf{w}_{i^{\prime} j^{\prime}}\right) \mathbf{w}_{i j},
$$

where $\Sigma^{\prime}$ means the sum over the required time and frequency intervals.

\section{B. Extension of method to incorporate other types of prior information}

A waveform reconstruction method more sophisticated than (4.4) can be obtained by generalizing the above analysis. Suppose that the prior PDF $p^{(0)}(\mathbf{h})$ is a general multivariate Gaussian in $\mathbf{h}$, such as

$$
p^{(0)}(\mathbf{h}) \propto \exp \left[-\frac{1}{2} \sum_{i j} \frac{\left(h_{i j}-\bar{h}_{i j}\right)^{2}}{\alpha_{i j}^{2}}\right],
$$

where $h^{i j}$ are the expansion coefficients of the signal $\mathbf{h}$ on some fixed wavelet basis $\mathbf{w}_{i j}$. By making suitable choices of the parameters $\bar{h}_{i j}$ and $\alpha_{i j}$, such a PDF could be chosen to encode the information that the frequency content of the signal at early times is concentrated near $f_{\text {merge }}$, that the signal joins smoothly onto the inspiral waveform, that at the end of merger the dominant frequency component is that of quasinormal ringing, etc. For any such prior PDF, it is straightforward to calculate the corresponding maximum likelihood estimator. If the prior PDF has expected value $\mathbf{h}_{0}$ and variancecovariance matrix $\boldsymbol{\Sigma}_{0}$, then the estimator is

$$
\mathbf{h}_{\text {best-fit }}(\mathbf{s})=\left[\boldsymbol{\Sigma}^{-1}+\boldsymbol{\Sigma}_{0}^{-1}\right]^{-1} \cdot\left[\boldsymbol{\Sigma}^{-1} \cdot \mathbf{s}+\boldsymbol{\Sigma}_{0}^{-1} \cdot \mathbf{h}_{0}\right] .
$$

Such an estimator could be calculated numerically.

\section{USING INFORMATION PROVIDED BY REPRESENTATIVE SUPERCOMPUTER SIMULATIONS}

In this section we propose a computational strategy for numerical relativists to pursue, if they successfully produce computer codes capable of simulating BBH mergers, but if running such codes is too expensive to permit an extensive survey of the merger parameter space. In this case, for data analysis purposes, it would be very useful to do a coarse survey of the BBH parameter space, with the aim of answering several qualitative questions and determining the range of several key parameters. Below we discuss several such issues, and describe how an understanding of them may impact data analysis.

One of the most important questions is, what is the approximate duration of the merger signal, and how does it depend on parameters such as the initial spins of the black holes and the mass ratio? The range of merger signal durations will be an important input to algorithms for searching for merger waves (the "noise-monitoring" technique described in Refs. [7, 42]) and algorithms for reconstructing the waveform from the data (see Sec. IV), particularly for cases in which the ringdown and/or inspiral signals are too weak to be seen in the data stream. Moreover, the duration of the waveform (together with its bandwidth) approximately determines the amount by which the SNR from band-pass filtering is lower than the matched filtering SNR obtained with merger templates [cf. Eq. (3.13)]. If it turns out that the duration is long (or, more relevantly, if $\mathcal{N}_{\text {bins }}$ is greater than our estimate of $\sim 30$ ), then the merger SNR will be badly degraded if templates are not available. Although the noisemonitoring technique will likely be useful for detecting merger waves, it will be difficult to reconstruct the waveform if $\mathcal{N}_{\text {bins }}$ is too large.

A similar question is the frequency bandwidth in which most of the merger waves' power is concentrated. In Ref. [7] we assumed that when one excises in the time domain the ringdown portion of the signal, the remaining signal has no significant power at frequencies above the quasi-normal ringing frequency of the final Kerr black hole. However, this assumption may not be valid; if it is not, signal searches and waveform reconstruction methods will need to incorporate this high-frequency power. As with the signal's duration, the range of bandwidths of merger waveforms will be an input to algorithms for reconstructing the merger waveform from the noisy data (see Sec. IV).

Another issue is how much energy is radiated in the merger compared to the energy radiated in the ringdown. Operationally, this question reduces to asking what proportion of the total waveform produced during the coalescence can be accurately fit by the ringdown's decaying sinusoid. In paper I we argued that if the spins of the black holes are large and aligned with one another and the orbital angular momentum, then the system has too much angular momentum for it to be lost solely through the ringdown, so that ringdown waves should not dominate the merger. On the other hand, if the spins of the black holes are small or not aligned, most of the radiated energy might well come out in ringdown waves. It may turn out that the ratio of energy radiated in the merger to that in the ringdown is small for all but a small set of merger parameters, which could have a great influence on BBH event searches.

It would be useful to know if the waveforms contain a strong signature of an "innermost stable circular orbit" (ISCO) $[8,9,43]$, as has commonly been assumed. In the extreme mass ratio limit $\mu \ll M$, there is such an orbit; when the smaller black hole reaches it, there is a sharp drop in the radiated energy per unit logarithmic frequency $d E / d \ln f$ [44]. However, there may not be such a sharp feature in the $d E / d \ln f$ plot in the equal-mass case, especially if the time scale over which the orbital instability operates is comparable to the radiation reaction time scale. 
Finally, it would be useful to know how much of the merger can be described as higher order quasi-normal ringing (QNR) modes. By convention, we have been calling that phase of the coalescence which is dominated by the most slowly damped, $l=m=2$ mode the ringdown phase, but before this mode dominates, QNR modes with different values of $l$ and/or $m$ are likely to be present. After the merger has evolved to the point when the merged object can be accurately described as a linear perturbation about a stationary black hole background, there might or might not be any significant subsequent period of time before the higher order modes have decayed away so much as to be undetectable. If simulations predict that higher order QNR modes are strong for a significant period of time, then these higher order QNR modes should be found by the normal ringdown search of the data stream; no extra search should be needed.

\section{ACCURACY REQUIREMENTS FOR MERGER WAVEFORM TEMPLATES}

For the remainder of the paper, we consider data analysis of merger waves using supercomputer templates. These templates will unavoidably contain numerical errors: if the physical waveform for some source is $h(t ; \boldsymbol{\theta})$, where the components of $\boldsymbol{\theta}=\left(\theta^{1}, \ldots, \theta^{n} p\right)$ are the various parameters upon which the waveform depends, then numerical simulations will predict the waveform $h(t ; \boldsymbol{\theta})+\delta h(t ; \boldsymbol{\theta})$. One would like the numerical error $\delta h(t ; \boldsymbol{\theta})$ to be small enough not to have a significant effect on signal searches, parameter extraction or any other types of data analysis that might be carried out using the template waveforms. In this section we suggest an approximate rule of thumb [Eq. (6.2)] for estimating when numerical errors are sufficiently small, and discuss its meaning and derivation.

\section{A. Accuracy criterion and implementation}

The accuracy criterion can be simply expressed in terms of the inner product introduced in Sec. II above [which is defined by Eq. (2.3) or alternatively by Eqs. (2.10)-(2.13)]: for a given template $h(t)$, our rule of thumb is that the numerical error $\delta h(t)$ should be small enough that the quantity

$$
\Delta \equiv \frac{1}{2} \frac{(\delta h \mid \delta h)}{(h \mid h)}
$$

satisfies

$$
\Delta \lesssim 0.01 \text {. }
$$

(The fractional loss in event detection rate in signal searches is $\sim 3 \Delta$, and so the value 0.01 corresponds to a $3 \%$ loss in event rate; see Sec. VI B.) If the errors at each data point $h_{j}=h\left(t_{j}\right)$ are uncorrelated, then Eq. (6.2) translates into a fractional accuracy for each data point of about $0.01 / \sqrt{\mathcal{N}_{\text {bins }}}$. If the errors add coherently in the integral (6.1), the fractional accuracy requirement will be more stringent.

It should be straightforward in principle to ensure that numerical templates satisfy Eq. (6.2). Let us schematically denote a numerically generated template as $h_{\text {num }}(t, \varepsilon)$, where $\varepsilon$ represents the set of tolerances (grid size, size of time steps, etc.) that govern the accuracy of the calculation. (Rep- resenting this set of parameters by a single tolerance $\varepsilon$ is an oversimplification but is adequate for the purposes of our discussion.) One can then iterate one's calculations varying $\varepsilon$ in order to obtain sufficiently accurate templates, using the following standard type of procedure: First, calculate the template $h_{\text {num }}(t, \varepsilon)$. Second, calculate the more accurate template $h_{\text {num }}(t, \varepsilon / 2)$. Third, make the identifications

$$
\begin{aligned}
h(t) & \equiv h_{\text {num }}(t, \varepsilon / 2), \\
\delta h(t) & \equiv h_{\text {num }}(t, \varepsilon / 2)-h_{\text {num }}(t, \varepsilon),
\end{aligned}
$$

and insert these quantities in Eq. (6.1) to calculate $\Delta$. This allows one to assess the accuracy of the template $h_{\text {num }}(t, \varepsilon)$. Finally, iterate until Eq. (6.2) is satisfied.

\section{B. Derivation and meaning of accuracy criterion}

The required accuracy of numerical templates depends on how and for what purpose they are used. As discussed in the Introduction, merger templates might be used in several different ways: (i) They might be used as search templates for signal searches using matched filtering. Such searches will probably not be feasible, at least initially, as they would require the computation of an inordinately large number of templates. (ii) For BBH events that have already been detected via matched filtering of the inspiral or ringdown waves, or by the noise-monitoring detection technique $[7,42]$ applied to the merger waves, the merger templates might be used for matched filtering in order to measure the binary's parameters and test general relativity. (iii) If only a few, representative supercomputer simulations and their associated waveform templates are available, one might simply perform a qualitative comparison between the measured waveform and templates in order to deduce qualitative information about the $\mathrm{BBH}$ source. In this section we estimate the accuracy requirements for the first two of these uses of merger templates.

Consider first signal searches using matched filtering. The expected SNR $\rho$ obtained for a waveform $h(t)$ when using a template $h_{T}(t)$ is [45]

$$
\rho=\frac{\left(h \mid h_{T}\right)}{\sqrt{\left(h_{T} \mid h_{T}\right)}} .
$$

Substituting $h_{T}(t)=h(t)+\delta h(t)$ into Eq. (6.4) and expanding to second order in $\delta h$, we find that the fractional loss in SNR produced by the numerical error $\delta h(t)$ is

$$
\frac{\delta \rho}{\rho}=\Delta_{1}+O\left[(\delta h)^{3}\right],
$$

where

$$
\Delta_{1} \equiv \frac{1}{2}\left[\frac{(\delta h \mid \delta h)}{(h \mid h)}-\frac{(\delta h \mid h)^{2}}{(h \mid h)^{2}}\right]
$$

Note that the quantity $\Delta_{1}$ is proportional to $\left(\delta h_{1} \mid \delta h_{1}\right)$, where $\delta h_{1}$ is the component of $\delta h$ perpendicular to $h$. Thus, a numerical error of the form $\delta h(t) \propto h(t)$ will not contribute to the fractional loss in SNR. This is to be expected, 
since the quantity (6.4) is independent of the absolute normalization of the templates $h_{T}(t)$.

The event detection rate is proportional to the cube of the SNR, and hence the fractional loss in event rate resulting from template inaccuracies is approximately $3 \delta \rho / \rho$ [45]. If one demands that the fractional loss in event rate be less than, say, $3 \%$ one obtains the criterion [46]

$$
\Delta_{1} \leqslant 0.01
$$

From Eqs. (6.1) and (6.6), $\Delta_{1} \leqslant \Delta$, and so the condition (6.7) is less stringent than the condition (6.2). The justification for imposing the more stringent criterion (6.2) rather than (6.7) derives from the use of templates for parameter extraction. We now turn to a discussion of this issue.

In principle, one could hope to measure all of the 15 parameters on which the merger waveforms depend by combining the outputs of several detectors with a complete bank of templates (although in practice the accuracy with which some of those 15 parameters can be measured is not likely to be very good). In the next few paragraphs we derive an approximate condition on $\Delta$ [Eq. (6.13)] which results from demanding that the systematic errors in the measured values of all the parameters be small compared to the statistical errors due to detector noise. (We note that one would also like to use matched filtering to test general relativity with merger waves; the accuracy criterion that we derive for parameter measurement will also approximately apply to tests of general relativity.)

Recall that we write the waveform as $h(t ; \boldsymbol{\theta})$. Let $\hat{\theta}^{\alpha}, 1$ $\leqslant \alpha \leqslant n_{p}$, be the best-fit values of $\theta^{\alpha}$ given by the matchedfiltering process. The quantities $\hat{\theta}^{\alpha}$ depend on the detector noise and are thus random variables. In the high SNR limit, the variables $\hat{\theta}^{\alpha}$ have a multivariate Gaussian distribution with (see, e.g., Ref. [13])

$$
\left\langle\delta \hat{\theta}^{\alpha} \delta \hat{\theta}^{\beta}\right\rangle=\Sigma^{\alpha \beta}
$$

where $\delta \hat{\theta}^{\alpha} \equiv \hat{\theta}^{\alpha}-\left\langle\hat{\theta}^{\alpha}\right\rangle$ and the matrix $\Sigma^{\alpha \beta}$ is defined after Eq. (2.17). The systematic error $\Delta \theta^{\alpha}$ in the inferred values of the parameters $\theta^{\alpha}$ due to the template error $\delta h$ can be shown to be approximately

$$
\Delta \theta^{\alpha}=\Sigma^{\alpha \beta}\left(\frac{\partial h}{\partial \theta^{\beta}} \mid \delta h\right) .
$$

From Eqs. (6.8) and (6.9), in order to guarantee that the systematic error in each of the parameters is smaller than some number $\varepsilon$ times that parameter's statistical error, we must have

$$
\left\|\delta h_{\|}\right\|^{2} \equiv\left(\delta h_{\|} \mid \delta h_{\|}\right) \leqslant \varepsilon^{2} .
$$

Here $\delta h_{\|}$is the component of $\delta h$ parallel to the tangent space of the manifold of signals $\mathcal{S}$ discussed in Sec. II. It is given by

$$
\delta h_{\|}=\Sigma^{\alpha \beta}\left(\delta h \mid \frac{\partial h}{\partial \theta^{\alpha}}\right) \frac{\partial h}{\partial \theta^{\beta}} .
$$

The magnitude $\left\|\delta h_{\|}\right\|$depends on details of the number of parameters and on how the waveform $h(t, \boldsymbol{\theta})$ varies with these parameters. However, a strict upper bound is

$$
\left\|\delta h_{\|}\right\| \leqslant\|\delta h\| \text {. }
$$

If we combine Eqs. (6.1), (6.10) and (6.12), we obtain

$$
\Delta \leqslant \frac{\varepsilon^{2}}{2 \rho^{2}} .
$$

Inserting reasonable estimates for $\rho$ and $\varepsilon(\rho \simeq 7, \varepsilon \simeq 1)$ we recover the criterion (6.2) [47]. In Sec. VII we give an alternative derivation of Eq. (6.13) using information theory.

The value $\rho \simeq 7$ leading to the criterion (6.2) is appropriate for ground based interferometers [7]. However, much higher SNRs are expected for LISA; see, e.g., Ref. [7]. Thus, numerical templates used for testing relativity and measuring parameters with LISA data will have to be substantially more accurate than those used with data from ground-based instruments.

\section{NUMBER OF BITS OF INFORMATION OBTAINABLE FROM THE MERGER SIGNAL AND IMPLICATIONS FOR TEMPLATE CONSTRUCTION}

In information theory, a quantity called "information" (analogous to entropy) can be associated with any measurement process: it is simply the base 2 logarithm of the number of distinguishable measurement outcomes [26,27]. Equivalently, it is the number of bits required to store the knowledge gained from the measurement. In this section we specialize the notions of information theory to gravitational wave measurements, and estimate the number of bits of information which one can gain in different cases.

\section{A. Total information gain}

First consider the situation in which templates are unavailable. Suppose that our prior information describing the signal is that it lies inside some frequency band of length $\Delta f$ and inside some time interval of duration $T$. We denote by $I_{\text {total }}$ the base 2 logarithm of the number of waveforms $\mathbf{h}$ that are distinguishable by the measurement, that are compatible with our prior information, and that are compatible with our measurement of the detector output's magnitude $\rho(\mathbf{s})$ [48]. Note that the vast majority of these $2^{I_{\text {total }}}$ waveforms are completely irrelevant to BBH mergers: the merger signals are a small subset (the manifold $\mathcal{S}$ ) of all distinguishable waveforms with the above characteristics. The quantity $I_{\text {total }}$ characterizes the information gain in a measurement when we do not have prior information about which waveforms are relevant. Note also that $I_{\text {total }}$ quantifies the information gained from the measurement about the merger waveform shape, but in the absence of templates, we do not learn anything about the BBH source.

A precise definition of the total information gain $I_{\text {total }}$ is as follows. Let $T$ and $\Delta f$ be a priori upper bounds for the durations and bandwidths of merger signals, and let $V$ be the vector space of signals with duration $\leqslant T$ inside the relevant frequency band. This vector space $V$ has dimension $\mathcal{N}_{\text {bins }}$ $=2 T \Delta f$. Let $p^{(0)}(\mathbf{h})$ be the PDF describing our prior infor- 
mation about the gravitational-wave signal [49], and let $p(\mathbf{h} \mid \mathbf{s})$ denote the posterior PDF for $\mathbf{h}$ after the measurement, i.e., the PDF for $\mathbf{h}$ given that the detector output is $\mathbf{s}$. A standard Bayesian analysis shows that $p(\mathbf{h} \mid \mathbf{s})$ will be given by

$$
p(\mathbf{h} \mid \mathbf{s})=\mathcal{K} p^{(0)}(\mathbf{h}) \exp [-(\mathbf{s}-\mathbf{h} \mid \mathbf{s}-\mathbf{h}) / 2]
$$

where $\mathcal{K}$ is a normalization constant [16]. Finally, let $p[\mathbf{h} \mid \rho(\mathbf{s})]$ be the PDF of $\mathbf{h}$ given that the magnitude of $\mathbf{s}$ is $\rho(\mathbf{s})$. We define $I_{\text {total }}$ to be

$$
I_{\text {total }} \equiv \int d \mathbf{h} p(\mathbf{h} \mid \mathbf{s}) \log _{2}\left[\frac{p(\mathbf{h} \mid \mathbf{s})}{p[\mathbf{h} \mid \rho(\mathbf{s})]}\right]
$$

By this definition, $I_{\text {total }}$ is the relative information of the PDFs $p[\mathbf{h} \mid \rho(\mathbf{s})]$ and $p(\mathbf{h} \mid \mathbf{s})$ [27]. In Appendix B we show that the quantity (7.2) in fact represents the base 2 logarithm of the number of distinguishable wave shapes that could have been measured and that are compatible with the magnitude $\rho(\mathbf{s})$ of the data stream [48]. Thus, one learns $I_{\text {total }}$ bits of information about the waveform $\mathbf{h}$ when one goes from knowing only the magnitude $\rho(\mathbf{s})=\|\mathbf{s}\|$ of the detector output to knowing the actual detector output $\mathbf{s}$.

We also show in Appendix B that in the limit of no prior information other than $T$ and $\Delta f$, we have

$$
I_{\text {total }}=\frac{1}{2} \mathcal{N}_{\text {bins }} \log _{2}\left[\rho(\mathbf{s})^{2} / \mathcal{N}_{\text {bins }}\right]+O\left[\ln \mathcal{N}_{\text {bins }}\right]
$$

The formula (7.3) is valid in the limit of large $\mathcal{N}_{\text {bins }}$ for fixed $\rho(\mathbf{s})^{2} / \mathcal{N}_{\text {bins }}$, and moreover applies only when

$$
\rho(\mathbf{s})^{2} / \mathcal{N}_{\text {bins }}>1
$$

see below for further discussion of this point.

There is a simple and intuitive way to understand the result (7.3). Fix the gravitational waveform, $\mathbf{h}$, considered as a point in the $\mathcal{N}_{\text {bins }}$-dimensional Euclidean space $V$. What is measured is the detector output $\mathbf{s}=\mathbf{h}+\mathbf{n}$, whose location in $V$ is displaced from $\mathbf{h}$. The direction and magnitude of the displacement depend upon the particular instance of the noise n. However, if we average over an ensemble of noise realizations, the displacement due to the noise is in a random direction and has rms magnitude $\sqrt{\mathcal{N}_{\text {bins }}}$ (since on an appropriate basis each component of $\mathbf{n}$ has rms value 1). Therefore, all points $\left\{\mathbf{h}^{\prime}\right\}$ lying inside a hypersphere of radius $\sqrt{\mathcal{N}_{\text {bins }}}$ centered on $\mathbf{h}$ are effectively indistinguishable from each other. The volume of such a hypersphere is

$$
C_{\mathcal{N}_{\text {bins }}}\left(\sqrt{\mathcal{N}_{\text {bins }}}\right)^{\mathcal{N}_{\text {bins }}}
$$

where $C_{\mathcal{N}_{\text {bins }}}$ is a constant whose value is unimportant. When we measure a detector output $\mathbf{s}$ with magnitude $\rho(\mathbf{s})$, the set of signals $\mathbf{h}$ that could have given rise to an identical measured $\rho(\mathbf{s})$ will form a hypersphere of radius $\sim \rho(\mathbf{s})$ and volume

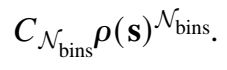

The number of distinguishable signals in this large hypersphere will be approximately the ratio of the two volumes (7.5) and (7.6); the base 2 logarithm of this ratio is the quantity (7.3).

Equation (7.3) expresses the information gain as a function of the magnitude of the measured detector output $\mathbf{s}$. We now re-express this information gain in terms of properties of the gravitational-wave signal $\mathbf{h}$. For a given $\mathbf{h}$, Eqs. (2.15) and (2.16) show that the detector output's magnitude $\rho(\mathbf{s})$ will be approximately given by

$$
\rho(\mathbf{s})^{2} \approx \rho^{2}+\mathcal{N}_{\text {bins }} \pm \sqrt{\mathcal{N}_{\text {bins }}}
$$

Here $\rho^{2}=\|\mathbf{h}\|^{2}$ is the SNR squared (2.4) that would be achieved if matched filtering were possible (if templates were available). We use $\rho$ as a convenient measure of signal strength; in this context, it is meaningful even in situations where templates are unavailable and matched filtering cannot be carried out. The last term in Eq. (7.7) gives the approximate size of the statistical fluctuations in $\rho(\mathbf{s})^{2}$. We now substitute Eq. (7.7) into Eq. (7.3) and obtain

$$
\begin{aligned}
I_{\text {total }}= & \frac{1}{2} \mathcal{N}_{\text {bins }} \log _{2}\left[1+\rho^{2} / \mathcal{N}_{\text {bins }}\right]\left[1+O\left(\frac{\ln \mathcal{N}_{\text {bins }}}{\mathcal{N}_{\text {bins }}}\right)\right. \\
& \left.+O\left(\frac{1}{\sqrt{\mathcal{N}_{\text {bins }}}}\right)\right] .
\end{aligned}
$$

Also, the condition (7.4) for the applicability of Eq. (7.3), when expressed in terms of $\rho$ instead of $\rho(\mathbf{s})$, becomes

$$
\frac{\rho^{2}}{\mathcal{N}_{\text {bins }}} \pm \frac{1}{\sqrt{\mathcal{N}_{\text {bins }}}} \geqslant 0
$$

which will be satisfied with high probability when $\rho$ $\gg \mathcal{N}_{\text {bins }}^{1 / 4}[50]$. In the regime $\rho \leqq \mathcal{N}_{\text {bins }}^{1 / 4}$, the condition (7.4) is typically not satisfied and the formula (7.3) does not apply; we show in Appendix B that in this case the information gain (7.2) is usually very small, depending somewhat on the prior PDF $p^{(0)}(\mathbf{h})$. [In contexts other than BBH merger waveforms, the information gain can be large in the regime $\rho$ $\ll \mathcal{N}_{\text {bins }}^{1 / 4}$ if the prior PDF $p^{(0)}(\mathbf{h})$ is very sharply peaked. For example, when one considers measurements of binary neutron star inspirals with advanced LIGO interferometers, the information gain in the measurement is large even though typically one will have $\rho \ll \mathcal{N}_{\text {bins }}^{1 / 4}$, because we have very good prior information about inspiral waveforms.]

As an example, a typical detected $\mathrm{BBH}$ event might have a merger SNR of $\rho \sim 10$, and $\mathcal{N}_{\text {bins }}$ might be 30 [7]. Then, Eq. (7.8) tells us that $\sim 3 \times 10^{9} \approx 2^{32}$ signals of the same magnitude could have been distinguished; thus the information gained is $\sim 32$ bits. More generally, for ground based interferometers we expect $\rho$ to lie in the range $5 \lesssim \rho \lesssim 100$ [7], and therefore 10 bits $\leq I_{\text {total }} \leqslant 120$ bits, and for LISA we expect $\rho$ to typically lie in the range $10^{3} \lesssim \rho \lesssim 10^{5}$ so that 200 bits $\lesssim I_{\text {total }} \lesssim 400$ bits.

\section{B. Source information gain}

Consider next the situation in which a complete family of accurate theoretical template waveforms $\mathbf{h}(\boldsymbol{\theta})$ is available for 
the merger. Without templates, we gain $I_{\text {total }}$ bits of information about the shape of the waveform in a measurement. With templates, some-but not all-of this information can be translated into information about the $\mathrm{BBH}$ source. For instance, suppose in the example considered above that the number of distinguishable waveforms that could have come from $\mathrm{BBH}$ mergers and that are distinguishable in the detector noise is $2^{25}$. (This number must be less that the total number $\sim 2^{32}$ of distinguishable waveform shapes, since waveforms from $\mathrm{BBH}$ mergers will clearly not fill out the entire function space $V$ of possible waveforms.) In this example, by identifying which template best fits the detector output, we can gain $\sim 25$ bits of information about the $\mathrm{BBH}$ source (e.g., about the black holes' masses or spins). We will call this number of bits of information $I_{\text {source }}$; clearly $I_{\text {source }}$ $\leqslant I_{\text {total }}$ always.

What of the remaining $I_{\text {total }}-I_{\text {source }}$ bits of information (7 bits in the above example)? If the detector output is close to one of the template shapes, this closeness can be regarded as evidence in favor of the theory of gravity (general relativity) used to compute the templates; so the $I_{\text {total }}-I_{\text {source }}$ bits can be viewed as information about the validity of general relativity. If one computed templates in more general theories of gravity, one could in principle translate those $I_{\text {total }}-I_{\text {source }}$ bits into a quantitative form and obtain constraints on the parameters entering into the gravitational theory. However, with only general-relativistic templates at one's disposal, the information contained in the $I_{\text {total }}-I_{\text {source }}$ bits will simply result in a qualitative confirmation of general relativity, in the sense that one of the general relativistic templates will fit the data well.

A precise definition of $I_{\text {source }}$ is as follows. Let $p(\boldsymbol{\theta} \mid \mathbf{s})$ denote the probability distribution for the source parameters $\boldsymbol{\theta}$ given the measurement $\mathbf{s}$. This PDF is given by a formula analogous to Eq. (7.1) [16]:

$$
p(\boldsymbol{\theta} \mid \mathbf{s})=\mathcal{K} p^{(0)}(\boldsymbol{\theta}) \exp [-(\mathbf{s}-\mathbf{h}(\boldsymbol{\theta}) \mid \mathbf{s}-\mathbf{h}(\boldsymbol{\theta})) / 2],
$$

where $p^{(0)}(\boldsymbol{\theta})$ is the prior PDF for $\boldsymbol{\theta}$ and $\mathcal{K}$ is a normalization constant. Let $p[\boldsymbol{\theta} \mid \rho(\mathbf{s})]$ be the posterior PDF for $\boldsymbol{\theta}$ given that the magnitude of the measured signal is $\rho(\mathbf{s})$. Then we define

$$
I_{\text {source }} \equiv \int d \boldsymbol{\theta} p(\boldsymbol{\theta} \mid \mathbf{s}) \log _{2}\left[\frac{p(\boldsymbol{\theta} \mid \mathbf{s})}{p[\boldsymbol{\theta} \mid \rho(\mathbf{s})]}\right]
$$

The number of bits (7.11) gained about the source will clearly depend on the details of how the gravitational waveforms depend on the source parameters, on the prior expected ranges of these parameters, etc. In Appendix B we argue that to a rather crude approximation, $I_{\text {source }}$ should be given by the formula (7.8) with $\mathcal{N}_{\text {bins }}$ replaced by the number of parameters $\mathcal{N}_{\text {param }}$ on which the waveform has a significant dependence:

$$
I_{\text {source }} \approx \frac{1}{2} \mathcal{N}_{\text {param }} \log _{2}\left[1+\rho^{2} / \mathcal{N}_{\text {param }}\right]
$$

Note that the quantity $\mathcal{N}_{\text {param }}$ should be bounded above by the quantity $n_{p}$ discussed in Sec. II, but may be somewhat smaller than $n_{p}$. This will be the case if the waveform depends only very weakly on some of the parameters $\theta^{\alpha}$. Equation (7.12) is only valid when $\mathcal{N}_{\text {param }} \leqslant \mathcal{N}_{\text {bins }}$. For BBH mergers we expect $\mathcal{N}_{\text {param }} \lesssim 15$, which from Eq. (7.12) predicts that $I_{\text {source }}$ lies in the range $\sim 10$ bits to $\sim 70$ bits for SNRs $\rho$ in the range 5-100 (the expected range for ground based interferometers [7]) and $\sim 100$ bits to $\sim 200$ bits for $\rho$ in the range $10^{3}-10^{5}$ expected for LISA [7].

\section{Loss of source information due to template inaccuracies or to sparseness in the lattice of templates}

As discussed in Sec. VI, templates will contain unavoidable numerical errors. We now analyze how such errors affect the source information gained, and use this analysis to infer the maximum allowable template error. We write

$$
\mathbf{h}_{T}(\boldsymbol{\theta})=\mathbf{h}(\boldsymbol{\theta})+\boldsymbol{\delta} \mathbf{h}(\boldsymbol{\theta}),
$$

where $\mathbf{h}(\boldsymbol{\theta})$ denotes the true waveform, $\mathbf{h}_{T}(\boldsymbol{\theta})$ the numerical template, and $\boldsymbol{\delta} \mathbf{h}(\boldsymbol{\theta})$ the numerical error. Clearly, the numerical error will reduce the information (7.11) one obtains about the source. To make an estimate of the reduction, we model the numerical error as a random process with

$$
\left\langle\delta h_{i} \delta h_{j}\right\rangle=\mathcal{C}_{i j}
$$

where for simplicity we take $\mathcal{C}_{i j}=\lambda \Gamma_{i j}$ for some constant $\lambda$. Here $\Gamma_{i j}$ is the matrix introduced in Eq. (2.10). The expected value of $(\delta h \mid \delta h)$ is then given by, from Eq. (2.13),

$$
\begin{aligned}
\langle(\delta h \mid \delta h)\rangle & =\Sigma^{i j}\left\langle\delta h_{i} \delta h_{j}\right\rangle \\
& =\Sigma^{i j} \lambda \Gamma_{i j}=\lambda \mathcal{N}_{\text {bins }},
\end{aligned}
$$

where we have used Eq. (2.9). We can write $\lambda$ in terms of the quantity $\Delta$ discussed in Sec. VI by combining Eqs. (6.1) and (7.15), yielding

$$
\lambda=2 \Delta \frac{\rho^{2}}{\mathcal{N}_{\text {bins }}} .
$$

The information $I_{\text {source }}^{\prime}$ which one obtains when measuring with inaccurate templates can be calculated by treating the sum of the detector noise $\mathbf{n}$ and the template numerical error $\boldsymbol{\delta} \mathbf{h}$ as an effective noise $\mathbf{n}^{\text {(eff) }}$. This effective noise is characterized by the covariance matrix

$$
\left\langle n_{i}^{(\mathrm{eff})} n_{j}^{(\mathrm{eff})}\right\rangle=\Gamma_{i j}+\lambda \Gamma_{i j} .
$$

Thus, in this simplified model, the effect of the numerical error is to increase the noise by a factor $\sqrt{1+\lambda}$. The new information gain $I_{\text {source }}^{\prime}$ is therefore given by Eq. (7.12) with $\rho$ replaced by an effective $\operatorname{SNR} \rho^{\prime}$, where

$$
\left(\rho^{\prime}\right)^{2}=\frac{\rho^{2}}{1+\lambda} .
$$

If we now combine Eqs. (7.12), (7.16) and (7.18), we find that the loss in information due to template inaccuracy,

$$
\delta I_{\text {source }}=I_{\text {source }}-I_{\text {source }}^{\prime},
$$


is given by

$$
\delta I_{\text {source }}=\rho^{2}\left(\frac{\rho^{2}}{\mathcal{N}_{\text {param }}+\rho^{2}}\right)\left(\frac{\mathcal{N}_{\text {param }}}{\mathcal{N}_{\text {bins }}}\right) \Delta+O\left(\Delta^{2}\right) .
$$

To ensure that $\delta I_{\text {source }} \lesssim 1$ bit, we therefore must have

$$
\Delta \lesssim \frac{1}{\rho^{2}}\left(\frac{\mathcal{N}_{\text {param }}+\rho^{2}}{\rho^{2}}\right)\left(\frac{\mathcal{N}_{\text {bins }}}{\mathcal{N}_{\text {param }}}\right) .
$$

This condition is a more accurate version of the condition (6.13) that was derived in Sec. VI. It approximately reduces to Eq. (6.13) for typical BBH events (except in the unrealistic limit $\left.\rho^{2} \ll \mathcal{N}_{\text {param }}\right)$, since $\mathcal{N}_{\text {param }} \sim 10$ and $10 \lesssim \mathcal{N}_{\text {bins }} \lessgtr 100$ [7].

Turn next to the issue of the required degree of fineness of a template lattice, that is, how close in parameter space successive templates must be to one another. We parameterize the fineness by a dimensionless parameter $\varepsilon_{\text {grid }}$ : the lattice is required to have the property that for any possible true signal $\mathbf{h}(\boldsymbol{\theta})$, there exists some template $\mathbf{h}\left(\boldsymbol{\theta}^{*}\right)$ in the lattice with

$$
\frac{\left(\mathbf{h}(\boldsymbol{\theta}) \mid \mathbf{h}\left(\boldsymbol{\theta}^{*}\right)\right)}{\sqrt{(\mathbf{h}(\boldsymbol{\theta}) \mid \mathbf{h}(\boldsymbol{\theta}))} \sqrt{\left(\mathbf{h}\left(\boldsymbol{\theta}^{*}\right) \mid \mathbf{h}\left(\boldsymbol{\theta}^{*}\right)\right)}} \geqslant 1-\varepsilon_{\text {grid }} .
$$

The quantity $1-\varepsilon_{\text {grid }}$ is called the minimal match [45]. Suppose that one defines a metric on the space $V$ of templates using the norm associated with the inner product (2.13). It then follows from Eq. (7.22) that the largest possible distance $D_{\max }$ between an incoming signal $\mathbf{h}(\boldsymbol{\theta})$ and some rescaled template $\mathcal{A h}\left(\boldsymbol{\theta}^{*}\right)$ with $\mathcal{A}>0$ is

$$
D_{\max }=\sqrt{2 \varepsilon_{\text {grid }}} \rho,
$$

where $\rho$ is the matched filtering SNR (2.4) of the incoming signal.

We can view the discreteness in the template lattice as roughly equivalent to an ignorance on our part about the location of the manifold $\mathcal{S}$ of true gravitational-wave signals between the lattice points. The maximum distance any correct waveform $\mathbf{h}(\boldsymbol{\theta})$ could be away from where we may think it should be (where our guess is for example obtained by linearly extrapolating from the nearest points on the lattice) is of order $D_{\max }$. We can crudely view this ignorance as equivalent to a numerical error $\delta \mathbf{h}$ in the templates of magnitude $\|\delta \mathbf{h}\|=D_{\max }$. Combining Eqs. (6.1), (7.20) and (7.23) shows that the loss of information $\delta I_{\text {source }}$ due to the discreteness of the grid should therefore be of order

$$
\delta I_{\text {source }} \sim \rho^{2}\left(\frac{\rho^{2}}{\mathcal{N}_{\text {param }}+\rho^{2}}\right)\left(\frac{\mathcal{N}_{\text {param }}}{\mathcal{N}_{\text {bins }}}\right) \varepsilon_{\text {grid }} .
$$

The grid fineness $\varepsilon_{\text {grid }}$ should be chosen to ensure that $\delta I_{\text {source }}$ is small compared to unity, while also taking into account that the fractional loss in event detection rate for signal searches due to the coarseness of the grid will be $\lesssim 3 \varepsilon_{\text {grid }}$; see Sec. VI B above and Refs. [45, 46]

\section{CONCLUSIONS}

Templates from numerical relativity for the merger phase of $\mathrm{BBH}$ coalescences will be a great aid to the analysis of detected BBH events. A complete bank of templates could be used to implement a matched filtering analysis of merger data, which would allow measurements of the binary's parameters and tests of general relativity in a strong field, highly dynamic regime. Such matched filtering may also be possible without a complete bank of templates, if iterative supercomputer simulations are carried out in tandem with data analysis. A match of the detected waves with such templates will be a triumph for the theory of general relativity and an unambiguous signature of the existence of black holes. Qualitative information from representative supercomputer simulations will also be useful, both as an input to algorithms for extracting the merger waveform's shape from the noisy interferometer data stream and as an aid to interpreting the observed waveforms and making deductions about the waves' source.

We have derived, using several rather different conceptual starting points, accuracy requirements that numerical templates must satisfy in order for them to be useful as data analysis tools. We first considered matched filtering signal searches using templates; here the loss in event rate due to template inaccuracies is simply related to the degradation in the SNR, and leads to a criterion on template accuracy. Approximately the same criterion is obtained when one demands that the systematic errors in parameter extraction be small compared to the detector-noise induced statistical errors. Finally, we quantified the information that is encoded in the merger waveforms using the framework of information theory, and deduced how much of the information is lost due to template inaccuracies or to having insufficiently many templates. We deduced approximate requirements that templates must satisfy (in terms both of individual template accuracy and of spacing between templates) in order that all of the waveform's information can be extracted.

The theory of maximum likelihood estimation is a useful starting point for deriving algorithms for reconstructing the gravitational waveforms from the noisy interferometer output. In this paper we have discussed and derived such algorithms in the contexts both of a single detector and of a network of several detectors; these algorithms can be tailored to build in many different kinds of prior information about the waveforms.

\section{ACKNOWLEDGMENTS}

We thank Kip Thorne for suggesting this project to us, and for his invaluable encouragement and detailed comments on the paper. We thank David Chernoff for some helpful conversations. This research was supported in part by NSF Grants PHY-9424337, PHY-9220644, and PHY-9514726, and NASA Grant NAGW-2897. S.A.H. gratefully acknowledges the support of the National Science Foundation Graduate program, and E.F. likewise acknowledges the support of Enrico Fermi and Sloan Foundations.

\section{APPENDIX A: WAVEFORM RECONSTRUCTION WITH A DETECTOR NETWORK}

In this appendix we describe how to extend the filtering methods discussed in Sec. IV above from a single detector to a network of an arbitrary number of detectors. The underly- 
ing principle is again simply to use the maximum likelihood estimator of the waveform shape. We also explain the relationship between our waveform reconstruction method and the method of Gürsel and Tinto [25]. Appendixes A 1 and A 2 below overlap somewhat with analyses by Finn [51]. Finn uses similar mathematical techniques to analyze measurements of a stochastic background and waves of wellunderstood form with multiple detectors, applications which are rather different from ours.

We start by establishing some notation for a detector network; these notation and conventions follow those of Appendix A of Ref. [13]. The output of such a network can be represented as a vector $\vec{s}(t)=\left[s_{1}(t), \ldots, s_{n_{d}}(t)\right]$, where $n_{d}$ is the number of detectors, and $s_{a}(t)$ is the strain amplitude read out from the $a$ th detector [52]. There will be two contributions to the detector output $\vec{s}(t)$-the detector network noise $\vec{n}(t)$ (a vector random process) and the true gravitational-wave signal $\vec{h}(t)$ :

$$
\vec{s}(t)=\vec{h}(t)+\vec{n}(t)
$$

We will assume that the detector network noise is stationary and Gaussian. This assumption is not very realistic, but understanding the optimal method of waveform reconstruction with this idealized assumptions is an important first step towards more sophisticated waveform reconstruction algorithms adapted to realistic detector noise. With this assumption, the statistical properties of the detector network noise can be described by the auto-correlation matrix

$$
C_{n}(\tau)_{a b}=\left\langle n_{a}(t+\tau) n_{b}(t)\right\rangle-\left\langle n_{a}(t+\tau)\right\rangle\left\langle n_{b}(t)\right\rangle,
$$

where the angular brackets mean an ensemble average or a time average. Twice the Fourier transform of the correlation matrix is the power spectral density matrix

$$
S_{h}(f)_{a b}=2 \int_{-\infty}^{\infty} d \tau e^{2 \pi i f \tau} C_{n}(\tau)_{a b} .
$$

The off-diagonal elements of this matrix describe the effects of correlations between the noise sources in the various detectors, while each diagonal element $S_{h}(f)_{a a}$ is just the usual power spectral density of the noise in the $a$ th detector. We assume that the functions $S_{h}(f)_{a b}$ for $a \neq b$ have been measured for each pair of detectors.

The Gaussian random process $\vec{n}(t)$ determines a natural inner product on the space of functions $\vec{h}(t)$, which generalizes the single-detector inner product (2.3). The inner product is defined so that the probability that the noise takes a specific value $\vec{n}_{0}(t)$ is

$$
p\left[\vec{n}=\vec{n}_{0}\right] \propto e^{-\left(\vec{n}_{0} \mid \vec{n}_{0}\right) / 2} .
$$

It is given by

$$
(\vec{g} \mid \vec{h}) \equiv 4 \operatorname{Re} \int_{0}^{\infty} d f \widetilde{g}_{a}(f) *\left[\mathbf{S}_{h}(f)^{-1}\right]^{a b} \widetilde{h}_{b}(f) .
$$

See, e.g., Appendix A of Ref. [13] for more details.
Turn, now, to the relation between the gravitational wave signal $h_{a}(t)$ seen in the $a$ th detector and the two independent polarization components $h_{+}(t)$ and $h_{\times}(t)$ of the waves. Let $\mathbf{x}_{a}$ be the position and $\mathbf{d}_{a}$ the polarization tensor of the $a$ th detector in the detector network. By polarization tensor we mean that tensor $\mathbf{d}_{a}$ for which the detector's output $h_{a}(t)$ is given in terms of the waves' transverse traceless strain tensor $\mathbf{h}(\mathbf{x}, t)$ by

$$
h_{a}(t)=\mathbf{d}_{a}: \mathbf{h}\left(\mathbf{x}_{a}, t\right),
$$

where the colon denotes a double contraction. A gravitational wave burst coming from the direction of a unit vector m will have the form

$$
\mathbf{h}(\mathbf{x}, t)=\sum_{A=+, \times} h_{A}(t+\mathbf{m} \cdot \mathbf{x}) \mathbf{e}_{\mathbf{m}}^{A},
$$

where $\mathbf{e}_{\mathbf{m}}^{+}$and $\mathbf{e}_{\mathbf{m}}^{\times}$are a basis for the transverse traceless tensors perpendicular to $\mathbf{m}$, normalized according to $\mathbf{e}_{\mathbf{m}}^{A}: \mathbf{e}_{\mathbf{m}}^{B}$ $=2 \delta^{A B}$. Combining Eqs. (A6) and (A7) and switching to the frequency domain using the convention (2.1) yields

$$
\widetilde{h}_{a}(f)=F_{a}^{A}(\mathbf{m}) \widetilde{h}_{A}(f) e^{-2 \pi i f \tau_{a}(\mathbf{m})},
$$

where the quantities

$$
F_{a}^{A}(\mathbf{m}) \equiv \mathbf{e}_{\mathbf{m}}^{A}: \mathbf{d}_{a},
$$

for $A=+, \times$, are detector beam-pattern functions for the $a$ th detector [34] and $\tau_{a}(\mathbf{m}) \equiv \mathbf{m} \cdot \mathbf{x}_{a}$ is the time delay at the $a$ th detector relative to the origin of coordinates.

\section{Derivation of posterior probability distribution}

We now construct the PDF $\mathcal{P}\left[\mathbf{m}, h_{+}(t), h_{\times}(t) \mid \vec{s}(t)\right]$ for the gravitational waves to be coming from direction $\mathbf{m}$ with waveforms $h_{+}(t)$ and $h_{\times}(t)$, given that the output of the detector network is $\vec{s}(t)$. Let $p^{(0)}(\mathbf{m})$ and $p^{(0)}\left[h_{A}(t)\right]$ be the prior probability distributions for the sky position $\mathbf{m}$ (presumably a uniform distribution on the unit sphere) and waveform shapes $h_{A}(t)$, respectively. A standard Bayesian analysis along the lines of that given in Ref. [16] and using Eq. (A4) gives

$$
\begin{aligned}
\mathcal{P}\left[\mathbf{m}, h_{A}(t) \mid \vec{s}(t)\right]= & \mathcal{K} p^{(0)}(\mathbf{m}) p^{(0)}\left[h_{A}(t)\right] \\
& \times \exp [-(\vec{s}-\vec{h} \mid \vec{s}-\vec{h}) / 2],
\end{aligned}
$$

where $\mathcal{K}$ is a normalization constant and $\vec{h}$ is understood to be the function of $\mathbf{m}$ and $h_{A}(t)$ given by (the Fourier transform of) Eq. (A8).

We next simplify Eq. (A10) by reducing the argument of the exponential from a double sum over detectors to a single sum over detector sites. In the next few paragraphs we carry out this reduction, leading to Eqs. (A18) and (A19) below. We assume that each pair of detectors in the network comes in one of two categories: (i) pairs of detectors at the same detector site, which are oriented the same way, and thus share common detector beam pattern functions $F_{a}^{A}(\mathbf{m})$ (for example the $2 \mathrm{~km}$ and $4 \mathrm{~km}$ interferometers at the LIGO Hanford site), or (ii) pairs of detectors at widely separated 
sites, for which the detector noise is effectively uncorrelated. Under this assumption we can arrange for the matrix $\mathbf{S}_{h}(f)$ to have a block diagonal form, with each block corresponding to a detector site, by choosing a suitable ordering of detectors in the list $\left(1, \ldots, n_{d}\right)$. Let us denote the detector sites by Greek indices $\alpha, \beta, \gamma \ldots$, so that $\alpha$ runs from 1 to $n_{s}$, where $n_{s}$ is the number of sites. Let $\mathcal{D}_{\alpha}$ be the subset of the detector list $\left(1, \ldots, n_{d}\right)$ containing the detectors at the $\alpha$ th site, so that any sum over detectors can be re-written

$$
\sum_{a=1}^{n_{d}}=\sum_{\alpha=1}^{n_{s}} \sum_{a \in \mathcal{D}_{\alpha}}
$$

Thus, for example, for a 3 detector network with 2 detectors at the first site and 1 at the second, $\mathcal{D}_{1}=\{1,2\}$ and $\mathcal{D}_{2}$ $=\{3\}$. Let $F_{\alpha}^{A}(\mathbf{m})$ denote the common value of the beam pattern functions (A9) for all the detectors at site $\alpha$. Let $\mathbf{S}_{\alpha}(f)$ denote the $\alpha$ th diagonal sub-block of the matrix $\mathbf{S}_{h}(f)$. Then if we define

$$
\Lambda=(\vec{s}-\vec{h} \mid \vec{s}-\vec{h})
$$

[the quantity which appears in the exponential in Eq. (A10)], we obtain, from Eq. (A5),

$$
\begin{aligned}
\Lambda= & \sum_{\alpha=1}^{n_{s}} 4 \operatorname{Re} \int_{0}^{\infty} d f \sum_{a, b \in \mathcal{D}_{\alpha}}\left[\widetilde{s}_{a}(f)^{*}-\widetilde{h}_{a}(f)^{*}\right] \\
& \times\left[\mathbf{S}_{\alpha}(f)^{-1}\right]^{a b}\left[\widetilde{s_{b}}(f)-\widetilde{h}_{b}(f)\right] .
\end{aligned}
$$

Next, we note from Eq. (A8) that the value of $\widetilde{h}_{a}$ will be the same for all detectors at a given site $\alpha$. If we denote this common value by $\widetilde{h}_{\alpha}$, then we obtain, after some manipulation of Eq. (A13),

$$
\Lambda=\sum_{\alpha=1}^{n_{s}} 4 \operatorname{Re} \int_{0}^{\infty} d f\left\{\frac{\left|\tilde{s}_{\alpha}(f)-\tilde{h}_{\alpha}(f)\right|^{2}}{S_{\alpha}^{(\mathrm{eff})}(f)}+\Delta_{\alpha}(f)\right\}
$$

The meanings of the various symbols in Eq. (A14) are as follows. The quantity $S_{\alpha}^{(\text {eff })}(f)$ is defined by

$$
\frac{1}{S_{\alpha}^{(\mathrm{eff})}(f)} \equiv \sum_{a, b \in \mathcal{D}_{\alpha}}\left[\mathbf{S}_{\alpha}(f)^{-1}\right]^{a b}
$$

and can be interpreted as the effective overall noise spectrum for site $\alpha$ [53]. The quantity $s_{\alpha}$ is given by

$$
\widetilde{s}_{\alpha}(f) \equiv S_{\alpha}^{(\text {eff })}(f) \sum_{a, b \in \mathcal{D}_{\alpha}}\left[\mathbf{S}_{\alpha}(f)^{-1}\right]^{a b} \widetilde{s_{b}}(f)
$$

and is, roughly speaking, the mean output strain amplitude of site $\alpha$. Finally,

$$
\begin{aligned}
\Delta_{\alpha}(f) \equiv & \sum_{a, b \in \mathcal{D}_{\alpha}} \widetilde{s_{a}}(f)^{*} \widetilde{s}_{b}(f)\left\{\left[\mathbf{S}_{\alpha}(f)^{-1}\right]^{a b}-S_{\alpha}^{(\mathrm{eff})}(f)\right. \\
& \left.\times \sum_{c, d \in \mathcal{D}_{\alpha}}\left[\mathbf{S}_{\alpha}(f)^{-1}\right]^{a c}\left[\mathbf{S}_{\alpha}(f)^{-1}\right]^{d b}\right\}
\end{aligned}
$$

The quantity $\Delta_{\alpha}$ is independent of $\mathbf{m}$ and $h_{A}(t)$, and is therefore irrelevant for our purposes; it can be absorbed into the normalization constant $\mathcal{K}$ in Eq. (A10). This unimportance of $\Delta_{\alpha}$ occurs because we are assuming that there is some signal present. The term $\Delta_{\alpha}$ is very important, however, in situations where one is trying to assess the probability that some signal (and not just noise) is present in the outputs of the detector network. In effect, it encodes the discriminating power against noise bursts which is due to the presence of detectors with different noise spectra at one site (e.g., the 2 $\mathrm{km}$ and $4 \mathrm{~km}$ interferometers at the LIGO Hanford site). We drop the term $\Delta_{\alpha}$ from now on.

The probability distribution for the waveform shapes and sky direction is now given by, from Eqs. (A10), (A12) and (A14),

$$
\mathcal{P}\left[\mathbf{m}, h_{A}(t) \mid \vec{s}(t)\right]=\mathcal{K} p^{(0)}(\mathbf{m}) p^{(0)}\left[h_{A}(t)\right] e^{-\Lambda^{\prime} / 2},
$$

where

$$
\Lambda^{\prime}=\sum_{\alpha=1}^{n_{s}} 4 \operatorname{Re} \int_{0}^{\infty} d f \frac{\left|\widetilde{s}_{\alpha}(f)-\widetilde{h}_{\alpha}(f)\right|^{2}}{S_{\alpha}^{(\mathrm{eff})}(f)} .
$$

Finally, we express this probability distribution directly in terms of the waveforms $h_{+}(t)$ and $h_{\times}(t)$ by substituting Eq. (A8) into Eq. (A19), which gives

$$
\begin{aligned}
\Lambda^{\prime}= & 4 \operatorname{Re} \int_{0}^{\infty} d f\left\{\sum_{A, B=+, \times} \Theta^{A B}(f, \mathbf{m})\left[\widetilde{h}_{A}(f)^{*}-\widetilde{\hat{h}}_{A}(f)^{*}\right]\right. \\
& \left.\times\left[\widetilde{h}_{B}(f)-\widetilde{\hat{h}}_{B}(f)\right]+\mathcal{S}(f, \mathbf{m})\right\} .
\end{aligned}
$$

Here

$$
\begin{gathered}
\Theta^{A B}(f, \mathbf{m}) \equiv \sum_{\alpha=1}^{n_{s}} \frac{F_{\alpha}^{A}(\mathbf{m}) F_{\alpha}^{B}(\mathbf{m})}{S_{\alpha}^{(\mathrm{eff})}(f)} \\
\tilde{\hat{h}}_{A}(f) \equiv \Theta_{A B}(f, \mathbf{m}) \sum_{\alpha=1}^{n_{s}} F_{\alpha}^{B}(\mathbf{m}) \widetilde{s}_{\alpha}(f) e^{2 \pi i f \tau_{\alpha}(\mathbf{m})},
\end{gathered}
$$

where $\Theta_{A B}$ is the inverse matrix to $\Theta^{A B}$, and

$$
\mathcal{S}(f, \mathbf{m})=\sum_{\alpha}\left|\widetilde{s}_{\alpha}(f)\right|^{2}-\Theta^{A B} \widetilde{\hat{h}}_{A}(f) * \widetilde{\hat{h}}_{B}(f) .
$$

\section{Estimating the waveform shapes and the direction to the source}

Equations (A18) and (A20) constitute one of the main results of this appendix, and give the final and general PDF for $\mathbf{m}$ and $h_{A}(t)$. In the next few paragraphs we discuss its implications. As mentioned at the start of the appendix, we are primarily interested in situations where the direction $\mathbf{m}$ to the source is already known. However, as an aside, we now briefly consider the more general context where the direction to the source as well as the waveform shapes are unknown.

Starting from Eq. (A18), one could use either maximum likelihood estimators or so-called Bayes estimators 
$[13,54-56]$ to determine "best-guess" values of $\mathbf{m}$ and $h_{A}(t)$. Bayes estimators have significant advantages over maximum likelihood estimators but are typically much more difficult to compute, as explained in, for example, Appendix A of Ref. [13]. The Bayes estimator for the direction to the source will be given by first integrating Eq. (A18) over all waveform shapes, which yields

$$
\mathcal{P}[\mathbf{m} \mid \vec{s}(t)]=\mathcal{K} p^{(0)}(\mathbf{m}) \mathcal{D}(\mathbf{m}) \exp \left[-2 \int_{0}^{\infty} d f \mathcal{S}(f, \mathbf{m})\right],
$$

where $\mathcal{D}(\mathbf{m})$ is a determinant-type factor that is produced by integrating over the waveforms $h_{A}(t)$. This factor encodes the information that the detector network has greater sensitivity in some directions than in others and that, other things being equal, a signal is more likely to have come from a direction in which the network is more sensitive. The Bayes estimator of $\mathbf{m}$ is now obtained simply by calculating the expected value of $\mathbf{m}$ with respect to the probability distribution (A24). The simpler, maximum likelihood estimator of $\mathbf{m}$ is given by choosing the values of $\mathbf{m}$ [and of $h_{A}(t)$ ] which maximize the probability distribution (A18) or, equivalently, by minimizing the quantity

$$
\int_{0}^{\infty} d f \mathcal{S}(f, \mathbf{m})
$$

Let us denote this value of $\mathbf{m}$ by $\mathbf{m}_{\mathrm{ML}}(\vec{s})$. Note that the quantity (A25) encodes all information about time delays between the signals detected at the various detector sites; as is well known, directional information is obtained primarily through time delay information [54].

In Ref. [25], Gürsel and Tinto suggest a method of estimating $\mathbf{m}$ from $\vec{s}(t)$ for a network of three detectors. For white noise and for the special case of one detector per site, the Gürsel-Tinto estimator is the same as the maximum likelihood estimator $\mathbf{m}_{\mathrm{ML}}(\vec{s})$ just discussed, with one major modification: in Sec. V of Ref. [25], Gürsel and Tinto prescribe discarding those Fourier components of the data whose SNR is below a certain threshold as the first stage of calculating their estimator.

Turn, now, to the issue of estimating the waveform shapes $h_{+}(t)$ and $h_{\times}(t)$. In general situations where both $\mathbf{m}$ and $h_{A}(t)$ are unknown, the best way to proceed in principle would be to integrate the probability distribution (A18) over all solid angles $\mathbf{m}$ to obtain a reduced probability distribution $\mathcal{P}\left[h_{A}(t) \mid \vec{s}(t)\right]$ for the waveform shapes and to use this reduced probability distribution to make estimators of $h_{A}(t)$. However, such an integration cannot be performed analytically and would not be easy numerically; in practice simpler estimators will likely be used. One such simpler estimator is the maximum likelihood estimator of $h_{A}(t)$ obtained from Eq. (A18). In the limit of no prior information about the waveform shape when the PDF $p^{(0)}\left[h_{A}(t)\right]$ is very broad, this maximum likelihood estimator is simply $\hat{h}_{A}(t)$ evaluated at the value $\mathbf{m}_{\mathrm{ML}}(\vec{s})$ of $\mathbf{m}$ discussed above.

For BBH mergers, in many cases the direction $\mathbf{m}$ to the source will have been measured from the inspiral portion of the waveform, and thus for the purposes of estimating the merger waveform's shape, $\mathbf{m}$ can be regarded as known. The probability distribution for $h_{A}(t)$ given $\mathbf{m}$ and $\vec{s}(t)$ is, from Eq. (A18),

$$
\mathcal{P}\left[h_{A}(t) \mid \mathbf{m}, \vec{s}(t)\right]=\mathcal{K}^{\prime} p^{(0)}\left[h_{A}(t)\right] e^{-\Lambda^{\prime \prime} / 2} .
$$

Here $\mathcal{K}^{\prime}$ is a normalization constant, and $\Lambda^{\prime \prime}$ is given by Eq. (A20) with the term $\mathcal{S}(f, \mathbf{m})$ omitted. The maximum likelihood estimator of $h_{A}(t)$ obtained from this probability distribution in the limit of no prior information is again just $\hat{h}_{A}(t)$. The formula for the estimator $\hat{h}_{A}(t)$ given by Eqs. (A15), (A16), (A21) and (A22) is one of the key results of this appendix. It specifies the best-fit waveform shape as a unique function of the detector outputs $s_{a}(t)$ for any detector network.

\section{Incorporating prior information}

In Sec. IV, we suggested a method of reconstruction of the merger waveform shape, for a single detector, which incorporated assumed prior information about the waveform's properties. In this appendix, our discussion so far has neglected all prior information about the shape of the waveforms $h_{+}(t)$ and $h_{\times}(t)$. We now discuss waveform estimation for a detector network, incorporating prior information, for fixed sky direction $\mathbf{m}$.

With a few minor modifications, the entire discussion of Sec. IV can be applied to a detector network. First, the linear space $V$ should be taken to be the space of pairs of waveforms $\left\{h_{+}(t), h_{\times}(t)\right\}$, suitably discretized, so that the dimension of $V$ is $2 T^{\prime} / \Delta t$. Second, the inner product (2.13) must be replaced by a discrete version of the inner product

$$
\begin{aligned}
\left(\left\{h_{+}, h_{\times}\right\} \mid\left\{k_{+}, k_{\times}\right\}\right) \equiv & 4 \operatorname{Re} \int_{0}^{\infty} d f \Theta^{A B}(f, \mathbf{m}) \\
& \times{\widetilde{h_{A}}}_{(f)} *{\widetilde{k_{B}}}_{B}(f),
\end{aligned}
$$

since the inner product (A27) plays the same role in the probability distribution (A26) as the inner product (2.13) plays in the distribution (4.3). Third, the estimated waveforms $\left\{\hat{h}_{+}(t), \hat{h}_{\times}(t)\right\}$ given by Eq. (A22) take the place of the measured waveform $\mathbf{s}$ in Sec. IV, for the same reason. Fourth, the wavelet basis used to specify the prior information must be replaced by a basis of the form $\left\{w_{i j}^{+}(t), w_{k l}^{\times}(t)\right\}$, where $w_{i j}^{+}(t)$ is a wavelet basis of the type discussed in Sec. IV for the space of waveforms $h_{+}(t)$, and similarly for $w_{k l}^{\times}(t)$. The prior information about, for example, the assumed duration and bandwidths of the waveforms $h_{+}(t)$ and $h_{\times}(t)$ can then be represented exactly as in Sec. IV. With these modifications, the remainder of the analyses of Sec. IV apply directly to a network of detectors. Thus the "perpendicular projection" estimator (4.4) and the more general estimator (4.12) can both be applied to a network of detectors.

\section{Gürsel-Tinto waveform estimator}

As mentioned in Sec. IV above, Gürsel and Tinto have suggested an estimator of the waveforms $h_{+}(t)$ and $h_{\times}(t)$ for networks of three detector sites with one detector at each site when the direction $\mathbf{m}$ to the source is known [57]. In our 
notation, the construction of that estimator can be summarized as follows. First, assume that the estimator is some linear combination of the outputs of the independent detectors corrected for time delays:

$$
\widetilde{\hat{h}}_{A}^{(G T)}(f)=\sum_{\alpha=1}^{3} w_{A}^{\alpha}(\mathbf{m}) e^{2 \pi i f \tau_{\alpha}(\mathbf{m})}{\widetilde{s_{\alpha}}}_{\alpha}(f) .
$$

Here $\widetilde{\hat{h}}_{A}^{(G T)}$ is the Gürsel-Tinto ansatz for the estimator, and $w_{A}^{\alpha}$ are some arbitrary constants that depend on $\mathbf{m}$. [Since there is only one detector per site, we can neglect the distinction between the output $\widetilde{s}_{a}(f)$ of an individual detector and the output $\widetilde{s}_{\alpha}(f)$ of a detector site.] Next, demand that for a noise-free signal the estimator reduce to the true waveforms $h_{A}(t)$. From Eqs. (A1) and (A8) above, this requirement is equivalent to

$$
\sum_{\alpha=1}^{3} w_{A}^{\alpha}(\mathbf{m}) F_{\alpha}^{B}(\mathbf{m})=\delta_{A}^{B} .
$$

There is a two dimensional linear space of tensors $w_{A}^{\alpha}$ which satisfy Eq. (A29). Finally, choose $w_{A}^{\alpha}$ subject to Eq. (A29) to minimize the expected value with respect to the noise of the quantity

$$
\sum_{A=+, \times} \int d t\left|\hat{h}_{A}^{(G T)}(t)-h_{A}(t)\right|^{2},
$$

where $\hat{h}_{A}^{(G T)}(t)$ is given as a functional of $h_{A}(t)$ and the detector noise $n_{\alpha}(t)$ by Eqs. (A1), (A8) and (A28).

It is straightforward to show by a calculation using Lagrange multipliers that the resulting estimator is [58]

$$
\hat{h}_{A}^{(G T)}(t)=\hat{h}_{A}(t)
$$

In other words, the Gürsel-Tinto estimator coincides with the maximum likelihood estimators of $h_{+}(t)$ and $h_{\times}(t)$ discussed in this appendix in the case of little prior information. However, the estimators discussed here generalize the Gürsel-Tinto estimator by allowing an arbitrary number of detectors per site [with the effective output and effective noise spectrum of a site being given by Eqs. (A16) and (A15) above], by allowing an arbitrary number of sites and by allowing one to incorporate prior information about the waveform shapes.

\section{APPENDIX B: MEASURES OF INFORMATION}

In this appendix we substantiate the claims concerning information theory made in Sec. VII of the body of the paper. First, we argue that the concept of the "relative information" of two PDFs introduced in Eq. (7.2) has the interpretation we ascribed to it: it is the base 2 logarithm of the number of distinguishable measurement outcomes. Second, we derive the approximations (7.8) and (7.12).

Consider first the issue of ascribing to any measurement process a "number of bits of information gained" from that process, which corresponds to the base 2 logarithm of the number of distinguishable possible outcomes of the measurement. If $p^{(0)}(\mathbf{x})$ is the PDF for the measured quantities $\mathbf{x}$ $=\left(x^{1}, \ldots, x^{n}\right)$ before the measurement, and $p(\mathbf{x})$ is the corresponding PDF after the measurement, then the relative information of these two PDFs is defined to be [27]

$$
I=\int d^{n} \mathbf{x} p(\mathbf{x}) \log _{2}\left[\frac{p(\mathbf{x})}{p^{(0)}(\mathbf{x})}\right] .
$$

In simple examples, it is easy to see that the quantity (B1) reduces to the number of bits of information gained in the measurement. For instance, if $\mathbf{x}=\left(x^{1}\right)$ and the prior PDF $p^{(0)}$ constrains $x^{1}$ to lie in some range of size $X$, and if after the measurement $x^{1}$ is constrained to lie in a small interval of size $\Delta x$, then $I \approx \log _{2}(X / \Delta x)$, as one would expect. In addition, the quantity (B1) has the desirable feature that it is coordinate independent, i.e., that the same answer is obtained when one makes a nonlinear coordinate transformation on the manifold parameterized by $\left(x^{1}, \ldots, x^{n}\right)$ before evaluating Eq. (B1). For these reasons, in any measurement process, the quantity (B1) can be interpreted as the number of bits of information gained.

\section{Explicit formula for the total information}

As a foundation for deriving the approximate formula (7.8), we derive in this subsection an explicit formula [Eq. (B13)] for the total information gain (7.2) in a gravitational wave measurement. We shall use a basis of $V$ where the matrix (2.10) is unity, and for ease of notation we shall denote by $\mathcal{N}$ the quantity $\mathcal{N}_{\text {bins }}$.

First, we assume that the prior $\operatorname{PDF} p^{(0)}(\mathbf{h})$ appearing in Eq. (7.1) is a function only of $h=\rho(\mathbf{h})$. In other words, all directions in the vector space $V$ are taken to be, a priori, equally likely, when one measures distances and angles with the inner product (2.13). It would be more realistic to make such an assumption with respect to a noise-independent inner product like $\left(h_{1} \mid h_{2}\right) \equiv \int d t h_{1}(t) h_{2}(t)$, but if the noise spectrum $S_{h}(f)$ does not vary too rapidly within the bandwidth of interest, the distinction is not too important and our assumption will be fairly realistic. We write the prior PDF as [59]

$$
\begin{aligned}
p^{(0)}(\mathbf{h}) d^{\mathcal{N}} h & =\frac{2 \pi^{\mathcal{N} / 2}}{\Gamma(\mathcal{N} / 2)} h^{\mathcal{N}-1} p^{(0)}(h) d h \\
& \equiv \bar{p}^{(0)}(h) d h
\end{aligned}
$$

The quantity $\bar{p}^{(0)}(h) d h$ is the prior probability that the signal $\mathbf{h}$ will have an SNR $\rho(\mathbf{h})$ between $h$ and $h+d h$. The exact form of the PDF $\bar{p}^{(0)}(h)$ will not be too important for our calculations below. A moderately realistic choice is $\bar{p}^{(0)}(h)$ $\propto 1 / h^{3}$ with a cutoff at some $h_{1} \ll 1$. Note, however, that the choice $p^{(0)}(\mathbf{h})=1$ corresponding to $\bar{p}^{(0)}(h) \propto h^{\mathcal{N}-1}$ is very unrealistic. Below we shall assume that $\bar{p}^{(0)}(h)$ is independent of $\mathcal{N}$.

We next write Eq. (7.1) in a more explicit form. Without loss of generality we can take

$$
\mathbf{s}=\left(s^{1}, \ldots, s^{\mathcal{N}}\right)=(s, 0, \ldots, 0),
$$

where $s=\rho(\mathbf{s})$. Then, writing $(\mathbf{s} \mid \mathbf{h})=s h \cos \theta$ and using the useful identity 


$$
d^{\mathcal{N}} h=\frac{2 \pi^{(\mathcal{N}-1) / 2}}{\Gamma[(\mathcal{N}-1) / 2]} \sin (\theta)^{\mathcal{N}-2} h^{\mathcal{N}-1} d \theta d h
$$

we can write

$$
\begin{aligned}
p(\mathbf{h} \mid \mathbf{s}) d^{\mathcal{N}} h= & \mathcal{K}_{1} \bar{p}^{(0)}(h) \sin (\theta)^{\mathcal{N}-2} \\
& \times \exp \left[-\frac{1}{2}\left(s^{2}+h^{2}-2 s h \cos \theta\right)\right] d h d \theta,
\end{aligned}
$$

where $\mathcal{K}_{1}$ is a constant. If we define the function $F_{\mathcal{N}}(x)$ by

$$
F_{\mathcal{N}}(x) \equiv \frac{1}{2} \int_{0}^{\pi} d \theta \sin (\theta)^{\mathcal{N}-2} e^{x \cos \theta}
$$

then $\mathcal{K}_{1}$ is determined by the normalization condition

$$
1=2 \mathcal{K}_{1} \int_{0}^{\infty} d h e^{-\left(s^{2}+h^{2}\right) / 2} F_{\mathcal{N}}(s h) \bar{p}^{(0)}(h) .
$$

We next calculate the PDF $p[\mathbf{h} \mid \rho(\mathbf{s})]$ appearing in the denominator in Eq. (7.2). From Bayes's theorem, this PDF is given by

$$
p[\mathbf{h} \mid \rho(\mathbf{s})]=\mathcal{K} p^{(0)}(\mathbf{h}) p[\rho(\mathbf{s}) \mid \mathbf{h}],
$$

where $p[\rho(\mathbf{s}) \mid \mathbf{h}]$ is the PDF for $\rho(\mathbf{s})$ given that the gravitational wave signal is $\mathbf{h}$, and $\mathcal{K}$ is a normalization constant. Using the fact that $p(\mathbf{s} \mid \mathbf{h}) \propto \exp \left[-(\mathbf{s}-\mathbf{h})^{2}\right]$, we find using Eq. (B4) that

$$
\begin{aligned}
p(\mathbf{s} \mid \mathbf{h}) d^{\mathcal{N}} s= & \frac{2^{1-\mathcal{N} / 2}}{\sqrt{\pi} \Gamma[(\mathcal{N}-1) / 2]} \sin (\theta)^{\mathcal{N}-2} s^{\mathcal{N}-1} \\
& \times \exp \left[-\frac{1}{2}\left(s^{2}+h^{2}-2 s h \cos \theta\right)\right] d s d \theta .
\end{aligned}
$$

Integrating over $\theta$ now yields, from Eq. (B6),

$$
p[\rho(\mathbf{s})=s \mid \mathbf{h}] d s \propto s^{\mathcal{N}-1} e^{-\left(s^{2}+h^{2}\right) / 2} F_{\mathcal{N}}(s h) d s .
$$

Now combining Eqs. (B4), (B8), and (B10) yields

$$
\begin{aligned}
p[\mathbf{h} \mid \rho(\mathbf{s})] d^{\mathcal{N}} h= & \mathcal{K}_{2} \bar{p}^{(0)}(h) e^{-\left[\rho(\mathbf{s})^{2}+h^{2}\right] / 2} F_{\mathcal{N}}[\rho(\mathbf{s}) h] \\
& \times \sin (\theta)^{\mathcal{N}-2} d h d \theta,
\end{aligned}
$$

where from Eq. (B7) the normalization constant is given by

$$
\mathcal{K}_{2}=\frac{2 \Gamma(\mathcal{N} / 2)}{\sqrt{\pi} \Gamma[(\mathcal{N}-1) / 2]} \mathcal{K}_{1}
$$

We can now calculate the information $I_{\text {total }}$ by combining Eqs. (7.2), (B5), (B6), (B11), and (B12). The result is

$$
\begin{aligned}
I_{\text {total }}[\rho(\mathbf{s}), \mathcal{N}]= & -\int_{0}^{\infty} d h p^{(1)}(h) G_{\mathcal{N}}[\rho(\mathbf{s}) h] \\
& -\log _{2}\left[\frac{2 \Gamma(\mathcal{N} / 2)}{\sqrt{\pi} \Gamma[(\mathcal{N}-1) / 2]}\right],
\end{aligned}
$$

where

$$
G_{\mathcal{N}}(x) \equiv \frac{x F_{\mathcal{N}}^{\prime}(x)}{\ln 2 F_{\mathcal{N}}(x)}-\log _{2} F_{\mathcal{N}}(x)
$$

and

$$
p^{(1)}(h) \equiv 2 \mathcal{K}_{1} \bar{p}^{(0)}(h) e^{-\left[\rho(\mathbf{s})^{2}+h^{2}\right] / 2} F_{\mathcal{N}}[\rho(\mathbf{s}) h] .
$$

Equations (B7), (B6), and (B13)-(B15) now define explicitly the total information $I_{\text {total }}$ as a function of the parameters $\rho(\mathbf{s})$ and $\mathcal{N}$ and of the prior PDF $\bar{p}^{(0)}(h)$.

\section{Approximate formula for the total information}

We now derive the approximate formula (7.8) for the total information. Let $\rho_{b}^{2}=\rho(\mathbf{s})^{2} / \mathcal{N}$; we will consider the limit of large $\rho(\mathbf{s})$ and $\mathcal{N}$ but fixed $\rho_{b}$. Our analysis will divide into two cases, depending on whether $\rho_{b}>1$ or $\rho_{b} \leqslant 1$. We first consider the case $\rho_{b}>1$. In the large $\mathcal{N}$ limit the result for $\rho_{b}>1$ will be independent of the prior PDF $\bar{p}^{(0)}(h)$, which we assume has no dependence on $\mathcal{N}$.

The first term in Eq. (B13) is the expected value $\left\langle G_{\mathcal{N}}[\rho(\mathbf{s}) h]\right\rangle$ of $G_{\mathcal{N}}[\rho(\mathbf{s}) h]$ with respect to the PDF (B15). If we change the variable of integration in this term from $h$ to $u=h / \sqrt{\mathcal{N}}$, we find

$$
\begin{aligned}
\left\langle G_{\mathcal{N}}[\rho(\mathbf{s}) h]\right\rangle \propto & \int_{0}^{\infty} d u \bar{p}^{(0)}(\sqrt{\mathcal{N}} u) e^{-\mathcal{N}\left(\rho_{b}^{2}+u^{2}\right) / 2} \\
& \times F_{\mathcal{N}}\left(\mathcal{N}_{\rho_{b}} u\right) G_{\mathcal{N}}\left(\mathcal{N} \rho_{b} u\right) .
\end{aligned}
$$

From Eq. (B6) it is straightforward to show that, in the limit of large $\mathcal{N}$,

$$
F_{\mathcal{N}}\left(\mathcal{N}_{z}\right) \approx \frac{1}{2} e^{\mathcal{N} q\left(\theta_{c}\right)} \sqrt{\frac{2 \pi}{\mathcal{N}\left|q^{\prime \prime}\left(\theta_{c}\right)\right|}}
$$

for fixed $z$. Here $q(\theta)$ is the function

$$
q(\theta)=z \cos \theta+\ln \sin \theta,
$$

and $\theta_{c}=\theta_{c}(z)$ is the value of $\theta$ which maximizes the function $q(\theta)$, given implicitly by

$$
z \sin ^{2} \theta_{c}=\cos \theta_{c} \text {. }
$$

We similarly find that

$$
F_{\mathcal{N}}^{\prime}\left(\mathcal{N}_{z}\right) \approx \frac{1}{2} e^{\mathcal{N} q\left(\theta_{c}\right)} \sqrt{\frac{2 \pi}{\mathcal{N}\left|q^{\prime \prime}\left(\theta_{c}\right)\right|}} \cos \theta_{c}
$$

It is legitimate to use the approximations (B17) and (B20) in the integral (B16) since the value $u_{\max }\left(\mathcal{N}, \rho_{b}\right)$ of $u$ at which 
the PDF $p^{(1)}\left(\mathcal{N} \rho_{b} u\right)$ is a maximum approaches at large $\mathcal{N}$ a constant $u_{\text {max }}\left(\rho_{b}\right)$ which is independent of $\mathcal{N}$, as we show below.

Inserting the approximation (B17) into Eq. (B16) and identifying $z=\rho_{b} u$, we find that the PDF (B15) is proportional to

$$
\exp [\mathcal{N Q}(u)+O(1)]
$$

where

$$
\mathcal{Q}(u)=-\frac{1}{2}\left(\rho_{b}^{2}+u^{2}\right)+q\left(\theta_{c}\right)
$$

and $\theta_{c}=\theta_{c}(z)=\theta_{c}\left(\rho_{b} u\right)$. From Eqs. (B18) and (B19) one finds that $\mathcal{Q}$ has a local maximum at

$$
u=u_{\max }=\sqrt{\rho_{b}^{2}-1}
$$

at which point $\theta_{c}$ is given by $\sin \theta_{c}=1 / \rho_{b}$. The form of the PDF (B21) now shows that, at large $\mathcal{N}$,

$$
\left\langle G_{\mathcal{N}}\left(\mathcal{N} \rho_{b} u\right)\right\rangle \approx G_{\mathcal{N}}\left(\mathcal{N} \rho_{b} u_{\text {max }}\right) .
$$

Finally, if we combine Eqs. (B13), (B17)-(B20), (B23) and (B24) and use Stirling's formula to approximate the $\Gamma$ functions, we obtain Eq. (7.3).

Turn, next, to the case $\rho_{b}<1$. In this case the function $\mathcal{Q}$ does not have a local maximum, and the dominant contribution to the integral (B16) at large $\mathcal{N}$ comes from $h \sim O(1)$ [rather than from $h \sim \sqrt{\mathcal{N}}, u \sim O(1)$ as was the case above]. From Eq. (B6) we obtain the approximations

$$
F_{\mathcal{N}}(\sqrt{\mathcal{N}} w)=\sqrt{\frac{\pi}{2 \mathcal{N}}} e^{w^{2} / 2}[1+O(1 / \sqrt{\mathcal{N}})]
$$

and

$$
F_{\mathcal{N}}^{\prime}(\sqrt{\mathcal{N}} w)=\sqrt{\frac{\pi}{2}} \frac{w}{\mathcal{N}} e^{w^{2} / 2}[1+O(1 / \sqrt{\mathcal{N}})]
$$

which are valid for fixed $w$ at large $\mathcal{N}$. Using Eqs. (B25), (B26), and (B13)-(B15), and using Stirling's formula again we find that

$$
I_{\text {total }} \approx \frac{1}{2} \rho_{b}^{2} \frac{\int_{0}^{\infty} d h \bar{p}^{(0)}(h) \exp \left[-\left(1-\rho_{b}^{2}\right) h^{2} / 2\right] h^{2}}{\int_{0}^{\infty} d h \bar{p}^{(0)}(h) \exp \left[-\left(1-\rho_{b}^{2}\right) h^{2} / 2\right]} .
$$

For simplicity we now take $\bar{p}^{(0)}(h)$ to be a Gaussian centered at zero with width $h_{\text {prior }}^{2}$; this yields

$$
I_{\mathrm{total}} \approx \frac{1}{2}\left[\frac{\rho_{b}^{2} h_{\mathrm{prior}}^{2}}{1+\left(1-\rho_{b}^{2}\right) h_{\mathrm{prior}}^{2}}\right]
$$

From Eq. (7.7), the parameter $\rho_{b}$ is given by

$$
\rho_{b}^{2}=1+\frac{\rho^{2}}{\mathcal{N}_{\text {bins }}} \pm \frac{1}{\sqrt{\mathcal{N}_{\text {bins }}}}
$$

where the last term denotes the rms magnitude of the statistical fluctuations. Since we are assuming that $\rho_{b}<1$, it follows that $\rho_{b}^{2} \approx 1-1 / \sqrt{\mathcal{N}_{\text {bins }}}$, and therefore we obtain, from Eq. (B28),

$$
I_{\text {total }} \approx \frac{1}{2} \min \left[h_{\text {prior }}^{2}, \sqrt{\mathcal{N}_{\text {bins }}}\right] .
$$

Thus, if $h_{\text {prior }} \lessgtr 1$, the total information gain is $\lesssim 1$ also.

\section{Approximate formula for the source information}

We now turn to a discussion of the approximate formula (7.12) for the information (7.11) obtained about the source of the gravitational waves. In general, the measure of information (7.11) depends in a complex way on the prior PDF $p^{(0)}(\mathbf{h})$ and on how the waveform $\mathbf{h}(\boldsymbol{\theta})$ depends on the source parameters $\boldsymbol{\theta}$. We can evaluate the information $I_{\text {source }}$ explicitly in the simple and unrealistic model where the dependence on the source parameters $\boldsymbol{\theta}$ is linear and where there is little prior information. In this case the manifold of possible signals is a linear subspace (with dimension $\mathcal{N}_{\text {param }}$ ) of the linear space of all possible signals (which has dimension $\mathcal{N}$ ). The integral (7.11) then reduces to an integral analogous to Eq. (7.2), and we obtain the formula (7.12) in the same way as we obtained Eq. (7.8). The result (7.12) is clearly a very crude approximation, as the true manifold of merger signals is very curved and nonlinear. Nevertheless, it seems likely that the formula (7.12) will be valid for some effective number of parameters $\mathcal{N}_{\text {param }}$ that is not too much different from the true number of parameters on which the waveform depends.
[1] A. Abramovici, W. E. Althouse, R. W. P. Drever, Y. Gürsel, S. Kawamura, F. J. Raab, D. Shoemaker, L. Sievers, R. E. Spero, K. S. Thorne, R. E. Vogt, R. Weiss, S. E. Whitcomb, and M. E. Zucker, Science 256, 325 (1992).

[2] C. Bradaschia et al., Nucl. Instrum. Methods Phys. Res. A 289, 518 (1990); also in Gravitation: a Banff Summer Institute, edited by R. Mann and P. Wesson (World Scientific, Singapore, 1991).

[3] J. Hough et al., "GEO600, Proposal for a $600 \mathrm{~m}$ Laser-
Interferometric Gravitational Wave Antenna,' 1994 (unpublished).

[4] P. Bender et al., "LISA, Laser interferometer space antenna for gravitational wave measurements: ESA Assessment Study Report,"' 1994.

[5] J. Hough et al., in Gravitational Wave Experiments, proceedings of the Edoardo Amaldi Conference, edited by E. Coccio, G. Pizzella, and F. Ronga (World Scientific, Singapore, 1995), p. 50 . 
[6] P. Bender, I. Ciufolini, K. Danzmann, W. Folkner, J. Hough, D. Robertson, A. Rüdiger, M. Sandford, R. Schilling, B. Schutz, R. Stebbins, T. Summer, P. Touboul, S. Vitale, H. Ward, and W. Winkler, "LISA: Laser Interferometer Space Antenna for the detection and observation of gravitational waves," pre-phase A report, 1995 (unpublished).

[7] É. É. Flanagan and S. A. Hughes, preceding paper, Phys. Rev. D 57, 4535 (1997).

[8] L. E. Kidder, C. M. Will, and A. G. Wiseman, Phys. Rev. D 47, 3281 (1993); Class. Quantum Grav. 9, L125 (1992).

[9] G. B. Cook, Phys. Rev. D 50, 5025 (1994). See especially Fig. 3.

[10] For the purpose of this paper, we consider the "intermediate binary black hole regime" $6 M \lesssim r \lesssim 12 M$ discussed in Ref. [7] to be part of the inspiral epoch.

[11] More information about the Grand Challenge Alliance can be found at http://www.npac.syr.edu/projects/ bh/; see also Ref. [12] for a review.

[12] L. S. Finn, in proceedings of GR14, gr-qc/9603004.

[13] É. E. Flanagan and C. Cutler, Phys. Rev. D 49, 2658 (1994).

[14] K. D. Kokkotas, A. Królak, and G. Tsegas, Class. Quantum Grav. 11, 1901 (1994); P. Jaranowski, K. D. Kokkotas, A. Królak, and G. Tsegas, ibid. 13, 1279 (1996).

[15] E. Poisson and C. M. Will, Phys. Rev. D 52, 848 (1995).

[16] L. S. Finn, Phys. Rev. D 46, 5236 (1992). See also Ref. [40], which is an addendum to Ref. [17] that shows that the methods used to calculate measurement errors in Refs. [17] and [16] are equivalent.

[17] F. Echeverria, Phys. Rev. D 40, 3194 (1988).

[18] For quantitative analyses of parameter extraction accuracies with LISA see C. Cutler, Phys. Rev. D (to be published), gr-qc/9703068; M. Peterseim et al., Class. Quantum Grav. 14, 1507 (1997); O. Jennrich et al., ibid. 14, 1525 (1997).

[19] The accuracy with which the various waveform parameters will be measurable is currently known only to factors of the order of 2. Statistical errors in maximum likelihood estimators have been calculated in the high SNR approximation [28,1315] and more accurately using Monte Carlo simulations [39]. However, ultimately one should use estimators (for example Bayes estimators) which perform better than maximum likelihood estimators [13,54-56]. In addition, all analyses to date have neglected waveform modulation due to the spin-induced precession of the orbital plane, an effect which will be important for BBHs with rapidly spinning BHs $[60,61]$. Thus the true parameter-measurement accuracies are still somewhat uncertain.

[20] The fraction of events with ringdown SNR greater than some threshold $\rho_{\text {ring }}$ is $\sim \rho_{\text {insp }}^{3} /\left(\alpha \rho_{\text {ring }}\right)^{3}$, where $\rho_{\text {insp }}$ is the detection threshold for inspiral SNRs, and $\alpha$ is the ratio between the actual inspiral and ringdown SNRs. This assumes that all events are detected via their inspiral signals and thus have inspiral SNRs larger than $\rho_{\text {insp. }}$. Equations (B4), (B5), (B16) and (B17) of Ref. [7] imply that $\alpha \sim 30$ for initial and advanced LIGO interferometers for $M \leqq 50 M_{\odot}$. Taking $\rho_{\text {ring }}$ $=1$ and using the typically discussed inspiral SNR threshold of $\rho_{\text {insp }}=6$ [33], one obtains that the fraction is about $1 \%$.

[21] C. M. Will, Phys. Rev. D 50, 6058 (1994); see also Ref. [14].

[22] T Damour and G. Esposito-Farese, Phys. Rev. Lett. 70, 2220 (1993); T. Damour and K. Nordtvedt, Phys. Rev. D 48, 3436 (1993).

[23] J. Bekenstein, to appear in the proceedings of the Second Sa- kharov Conference in Physics, gr-qc/9605059.

[24] We discuss only Wiener optimal filtering, but note that there are more sophisticated nonlinear filtering methods [13,54-56] that could be used to give better results for parameter measurements (see Ref. [19]). The use of such methods would be more computationally intensive than linear filtering, but on the other hand, vast amounts of supercomputer time would have already been expended to generate merger templates.

[25] Y. Gürsel and M. Tinto, Phys. Rev. D 40, 3884 (1989).

[26] L. Brillouin, A Physical Theory of Information (IBM Watson Laboratories, New York, 1952).

[27] T. M. Cover and J. A. Thomas, Elements of Information Theory (Wiley, New York, 1991).

[28] L. S. Finn and D. F. Chernoff, Phys. Rev. D 47, 2198 (1993).

[29] Our convention for the normalization of the spectral noise density $S_{h}(f)$ is such that

$$
\left\langle n(t)^{2}\right\rangle=\int_{0}^{\infty} S_{h}(f) d f .
$$

[30] S. V. Dhurandhar and B. F. Schutz, Phys. Rev. D 50, 2390 (1994).

[31] Strictly speaking this is not a real basis, but a real basis could be obtained by taking $\mathbf{d}_{0}$ and the real and imaginary parts of $\mathbf{d}_{k}$ for $k>0$.

[32] When a complete set of theoretical templates is available so that matched filtering can be carried out, reconstructing the "best-fit" waveform is of course trivial: it is just the template which maximizes the signal-to-noise ratio.

[33] C. Cutler, T. A. Apostolatos, L. Bildsten, L. S. Finn, É. E. Flanagan, D. Kennefick, D. M. Marković, A. Ori, E. Poisson, G. J. Sussman, and K. S. Thorne, Phys. Rev. Lett. 70, 2984 (1993).

[34] K. S. Thorne, in 300 Years of Gravitation, edited by S. W. Hawking and W. Israel (Cambridge University Press, Cambridge, England, 1987), pp. 330-458.

[35] Contrary to one's intuition, the value (3.17) for advanced interferometers is lower than the value (3.16) for initial interferometers. This is because the advanced interferometers can detect inspirals with lower band-pass filtering SNRs than the initial interferometers, due to the larger number of cycles of the inspiral signal in the advanced interferometer's bandwidth.

[36] L. A. Wainstein and V. D. Zubakov, Extraction of Signals from Noise (Prentice-Hall, Englewood Cliffs, NJ, 1962).

[37] C. W. Helstrom, Statistical Theory of Signal Detection (Pergamon, Oxford, 1968).

[38] P. Jaranowski and A. Krolak, Phys. Rev. D 49, 1723 (1994); P. Jaranowski, K. D. Kokkotas, A. Krolak, and G. Tsegas, Class. Quantum Grav. 13, 1279 (1996).

[39] R. Balasubramanian, B. S. Sathyaprakash, and S. V. Dhurandhar, Phys. Rev. D 53, 3033 (1996); 54, 1860 (1996); R. Balasubramanian and S. V. Dhurandhar, ibid. 57, 3408 (1998); C. Cutler (unpublished).

[40] F. Echeverria, Ph.D. thesis, California Institute of Technology, 1993, addendum to Chap. 2.

[41] For example, one can first truncate the signal in the time domain, then perform a finite Fourier transform, and then throw away the Fourier coefficients outside the frequency range of interest. Or one can first band-pass filter the data stream, and then truncate in the time domain.

[42] É. É. Flanagan (in preparation).

[43] D. Lai and A. G. Wiseman, Phys. Rev. D 54, 3958 (1996).

[44] A. Ori and K. S. Thorne (in preparation). 
[45] B. J. Owen, Phys. Rev. D 53, 6749 (1996).

[46] In fact, for detection purposes, the criterion (6.7) is a little more stringent than need be: When one attempts to detect the signal, one computes the maximum overlap between the data stream and all possible templates, and thus the relevant quantity to calculate is [45]

$$
\max _{\Delta \boldsymbol{\theta}} \frac{\left(\mathbf{h}(\boldsymbol{\theta}) \mid \mathbf{h}_{T}(\boldsymbol{\theta}+\Delta \boldsymbol{\theta})\right)}{\sqrt{\left(\mathbf{h}_{T}(\boldsymbol{\theta}+\Delta \boldsymbol{\theta}) \mid \mathbf{h}_{T}(\boldsymbol{\theta}+\Delta \boldsymbol{\theta})\right)}},
$$

which is larger than

$$
\frac{\left(\mathbf{h}(\boldsymbol{\theta}) \mid \mathbf{h}_{T}(\boldsymbol{\theta})\right)}{\sqrt{\left(\mathbf{h}_{T}(\boldsymbol{\theta}) \mid \mathbf{h}_{T}(\boldsymbol{\theta})\right)}} .
$$

However, we neglect this effect here as it is not possible to calculate it in the absence of a model waveform $\mathbf{h}(\boldsymbol{\theta})$ for merger waves. For inspiral waves, relatively crude templates will suffice to detect the waves, and more accurate templates will be needed to measure parameters.

[47] Criterion (6.13) is probably rather more stringent than need be: the left hand side of Eq. (6.12) is likely smaller than the right hand side by a factor $\sim \sqrt{n_{p} / \mathcal{N}_{\text {bins }}}$, where $n_{p}$ is the number of parameters and $\mathcal{N}_{\text {bins }}$ is the dimension of the total space of signals $V$.

[48] No significant changes would result from changing the requirement "have the same magnitude as the measured signal" to "have a magnitude which is smaller than or equal to that of the measured signal"; the numerical value of the information would be about the same. The reason we insert this requirement into our definition of information [so that we use the PDF $p[\mathbf{h} \mid \rho(\mathbf{s})]$ in Eq. (7.2) rather than the PDF $\left.p^{(0)}(\mathbf{h})\right]$ is that the resulting notion of information is more useful and relevant than the notion obtained from using $p^{(0)}(\mathbf{h})$. If one were to use the PDF $p^{(0)}$, the resulting "information gain in the measurement" would be the same for all detected signals, whether the SNR be 10 or 1000 .

[49] The PDF $p^{(0)}(\mathbf{h})$ describes all one's prior information about the signal $\mathbf{h}$, except for the information that the maximum expected duration is $T$ and that the maximum expected bandwidth is $\Delta f$; that information is already encoded in the choice of the space $V$.

[50] Note that the regime $\rho \gg \mathcal{N}_{\text {bins }}^{1 / 4}$ coincides with the regime in which the signal is detectable using the noise-monitoring search method discussed in Refs. [7, 42].

[51] L. S. Finn (in preparation).

[52] Note that in Appendix A of Ref. [13], this quantity was denoted $\mathbf{s}(t)$ rather than $\vec{s}(t)$. Here we use the alternative notation $\vec{s}(t)$ since the notation $\mathbf{s}$ has already been used in Sec. IV to denote the vector $(2.5)$.

[53] The effective site noise $S_{\alpha}^{(\text {eff })}(f)$ is also the relevant quantity which governs the sensitivity of a given site to stochastic gravitational waves; see Eq. (3.13) of É. É. Flanagan, Phys. Rev. D 48, 2389 (1993).

[54] B. F. Schutz, in The Detection of Gravitational Radiation, edited by D. Blair (Cambridge University Press, Cambridge, England, 1989).

[55] M. H. A. Davis, in Gravitational Wave Data Analysis, edited by B. F. Schutz (Kluwer Academic, Boston, 1989).

[56] D. Nicholson and A. Vecchio, Phys. Rev. D 57, 4588 (1997).

[57] See Eqs. (4.17)-(4.22) and Appendix C of Ref. [25].

[58] In fact, this result is independent of the specific choice (A30) of norm on the space of waveform pairs $\left\{h_{+}(t), h_{\times}(t)\right\}$.

[59] In Eqs. (B2), (B4), (B5), (B9), and (B11) we omit factors of $\mathcal{N}-2$ dimensional solid angle elements $d \Omega^{\mathcal{N}-2} / C_{\mathcal{N}-2}$ which integrate to 1 . Thus, the equal symbol in these equations should be interpreted to mean that the PDFs on the left and right hand sides will produce identical expected values of functions that depend on $\theta$ and $h$ only.

[60] T. A. Apostolatos, C. Cutler, G. J. Sussman, and K. S. Thorne, Phys. Rev. D 49, 6274 (1994).

[61] L. E. Kidder, Phys. Rev. D 52, 821 (1995). 